\title{
HYPERSPECTRAL NEAR INFRARED ASSESSMENT OF THE BRAIN IN CARDIAC ARREST
}

\author{
by \\ Thu Nga Nguyen \\ Bachelor of Science, Ryerson University, 2016 \\ A thesis \\ presented to Ryerson University \\ in partial fulfillment of the requirements for the degree of \\ Master of Science \\ in the program of \\ Biomedical Physics
}

Toronto, Ontario, Canada, 2018

CThu Nga Nguyen, 2018 
I hereby declare that I am the sole author of this thesis. This is a true copy of the thesis, including any required final revisions, as accepted by my examiners.

I authorize Ryerson University to lend this thesis to other institutions of individuals for purpose of scholarly research.

I further authorize Ryerson University to reproduce this thesis by photocopying or by other means, in total or in part, at the request of other institutions or individuals for the purpose of scholarly research.

I understand that my thesis may be made electronically available to the public. 


\title{
Hyperspectral near infrared assessment of the brain in cardiac arrest
}

\author{
Master of Science, 2018 \\ Thu Nga Nguyen \\ Biomedical Physics \\ Ryerson University
}

\begin{abstract}
$\underline{\text { Abstract }}$
Two-thirds of out-of-hospital cardiac arrest patients, who survive to hospital admission, die in the hospital from neurological injuries related to cerebral hypoperfusion. Hyperspectral near infrared spectroscopy (hNIRS) is a non-invasive technique that measures the major chromophores in the brain, such as oxygenated hemoglobin, deoxygenated hemoglobin and cytochrome $\mathrm{C}$ oxidase (CCO), an intracellular marker of oxygen consumption. We have demonstrated that hNIRS can detect changes in cerebral oxygenation and metabolism in patients undergoing transcatheter aortic valve insertion (TAVI) - a procedure that temporarily induces sudden hypotension and hypoperfusion that mimics cardiac arrest. Using multi-distance hNIRS, we found that while measured regional oxygen saturation $(\mathrm{rSO} 2)$ changes resulted mainly from the extra-cerebral tissues, CCO changes during cardiac arrests occurred mainly in the brains of patients. We also applied the hNIRS algorithm based on the "2-layer model" to the data to measure cerebral oxygen saturation and $\mathrm{CCO}$ in patients during the procedure. We found that changes in all the parameters scale with the thickness of the extra-cerebral layer. Finally, the results of the comparison of breath holding measurement by using multispectral NIRS algorithm with the selected wavelength combinations from a previous study on animal suggests that changes in CCO could be measured, however wavelengths need to be further optimized for the adult patients.
\end{abstract}




\section{Acknowledgements}

I would like to thank my supervisor, Dr. Vladislav Toronov for allowing me this opportunity to be a part of this research experience. He has inspired me to work as an independent researcher and help me to understand the basic knowledge of my research. The door to his office was always open whenever I had a question about research or writing throughout the term. Thank you for your valuable guidance and encouragement and most importantly, the confidence to believe in myself for any challenges in the future.

I would like to thank my supervisory committee, Dr. Steve Lin and Dr. Jahan Tavakkoli for taking their time and giving me their suggestions and encouragement during those long research meetings. Thank you for taking time to provide me endless support and advice on my research. I would like to thank the group of graduate students. I will miss you and cherish all moments of graduate life together.

I also want to take this opportunity to express my gratitude to all the of members of the Physics department for their help and support.

Thank you to my family, especially my mother who is always there for me and has helped me to become the person I am today. 


\section{Dedication}

To my mother, a strong and wonderful woman who supported me and my brother with her unconditional love. 


\section{$\underline{\text { TABLE OF CONTENTS }}$}

Abstract............................................................................................................................................................ iii

Acknowledgements ................................................................................................................. iv

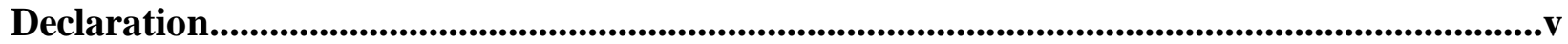

List of Tables .......................................................................................................................................... viii

List of Figures................................................................................................................................. ix

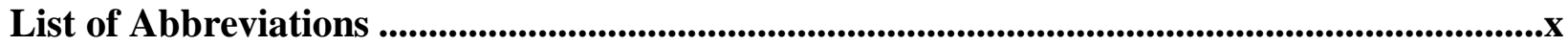

Chapter 1 - Introduction and background ......................................................................................1

1.1 - The physics of light transport in tissues ...........................................................................................2

1.2 - Near-infrared spectroscopy in the human brain ................................................................................

1.3 - The history of Near Infrared in the human brain

1.4 - Different NIRS spectroscopy measurements .......................................................................................5

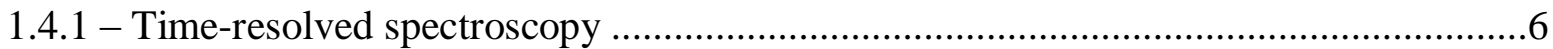

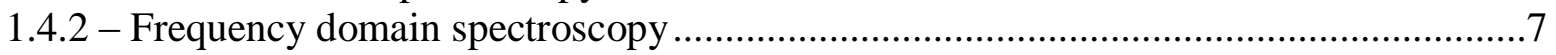

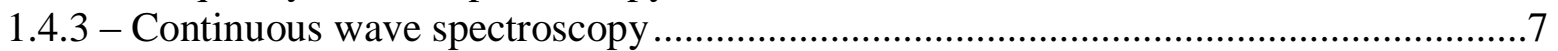

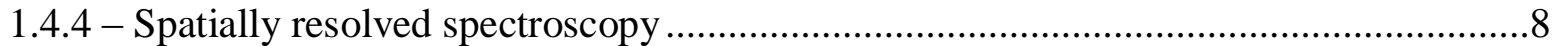

1.5 - Tissue Chromophores ..........................................................................................................................8

1.5.1 - Oxyhemoglobin and deoxyhemoglobin as oxygen transport agents ..........................

1.5.2 - Cytochrome $\mathrm{C}$ oxidase as an oxygen consume agent ...............................................11

1.6 - The clinical need for cerebral oxygenation measurement during cardiac arrest.....................16

1.7 - Basic anatomy of human head .......................................................................................................18

1.8 - NIRS and current two-layer model of the human head................................................................19

1.9 - Algorithms.......................................................................................................................................21

1.9.1 - Modified Beer-Lambert Law on the commercial multispectral NIRS .....................21

1.9.1.1 - The availability of multiple source-detector distances on the commercial

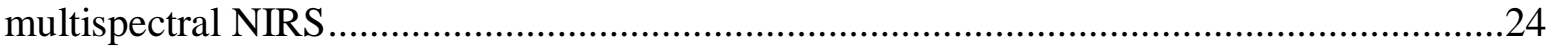

1.9.1.2 - Limitation of the modified Beer-Lambert Law algorithm ................................26

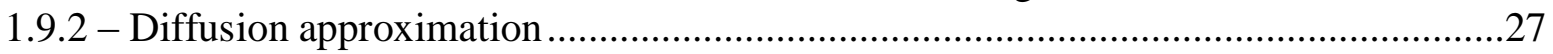

1.9.2.1 - Hyperspectral near-infrared spectroscopy algorithm using 2-layer model.........29

1.10 - Wavelength selection for hNIRS algorithm ................................................................................31

1.11 - Thesis organization and objectives ................................................................................................33

Chapter 2 - Journal Manuscript .................................................................................................36

Abstract ....................................................................................................................................................37

1 - Introduction .............................................................................................................................................37

2 - Materials and methods ............................................................................................................................40

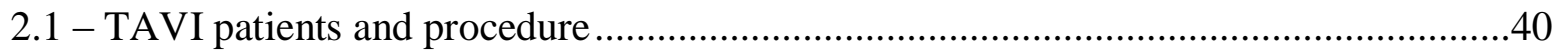

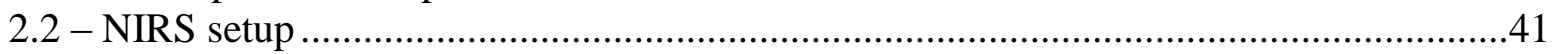

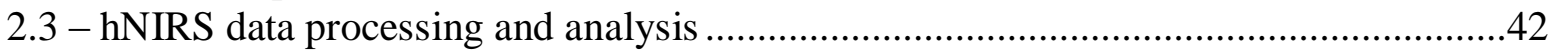

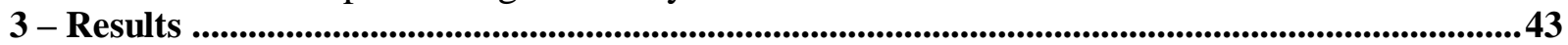

4 - Discussion .........................................................................................................................................................44

5 - Conclusion .........................................................................................................................................49

Acknowledgements..............................................................................................................................49

Chapter 3 - Additional Methods ….............................................................................................50

3.1 - Breath holding measurement ...................................................................................................50

3.1.1 - System overview and respiratory challenges ....................................................51

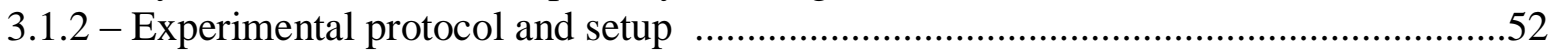




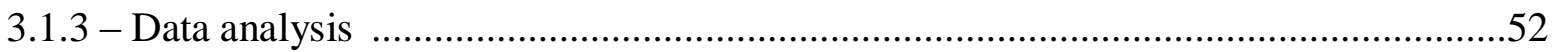

3.2 - Results and Discussion of 2 layer-model and breath holding measurements .......................54

3.2.1 - Results of 2 layer-model and breath holding measurements .................................50

3.2.2 - Discussion of 2 layer-model and breath holding measurements ............................50

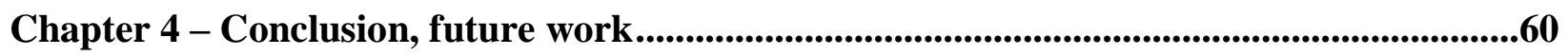

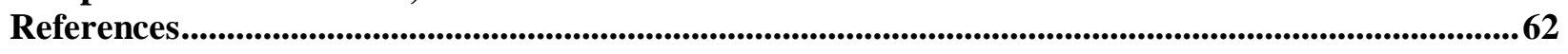




\section{$\underline{\text { LIST OF TABLES }}$}

Table 1.1. Near infrared spectroscopy monitoring during cardiac arrest. ..................................17

Table 1.2. Applications of cerebral measurements using mNIRS.

Table 2.1. The hNIRS-measured cerebral hemodynamics and metabolism for the $1 \mathrm{~cm}$ and $3 \mathrm{~cm}$ channels during the ventricular pacing for TAVI patients. The number of RVP episodes for each patient corresponds to the number of positive or negative response detections indicated as ' $\mathrm{Y}$ ' and ' $\mathrm{N}$ ', respectively. 


\section{LIST OF FIGURES}

Figure 1.1 Principle of light propagation in the brain by NIRS. ......................................... 3

Figure 1.2 Different techniques used in the field of Near Infrared Spectroscopy: Time-domain,

Frequency-domain, Continuous-wave and spatially-resolved NIRS.

Figure 1.3. The absorption spectra of oxyhemoglobin, deoxyhemoglobin and cytochrome c oxidase.

Figure 1.4. The schematic of cellular metabolism with electron transport chain and CCO

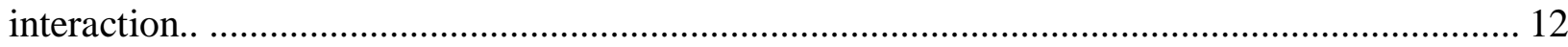

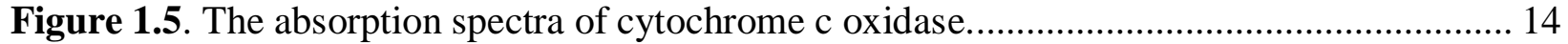

Figure 1.6. The anatomy of the human head....................................................................... 19

Figure 1.7. Schematic presentation of the brain in NIRS. ............................................... 20

Figure 1.8. Hyperspectral NIRS data processing algorithm for changes in the concentrations of

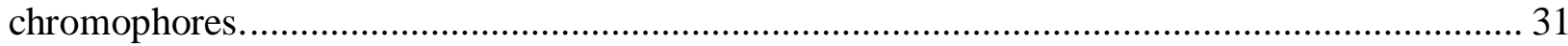

Figure 1.9. The gold standard values of the changes in concentrations of $\mathrm{HbO}_{2}, \mathrm{Hb}$, and $\mathrm{CCO}$

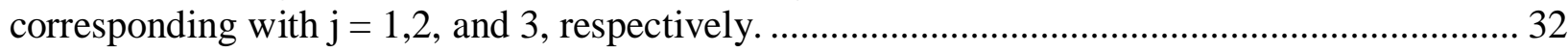

Figure 2.1. TAVI experimental setup. ..................................................................... 41

Figure 2.2. The time-course series of the changes in different parameters of a TAVI patient 10 (see Table 1) for $[\mathrm{Hb}]$ and $\left[\mathrm{HBO}_{2}\right]$ changes a) at the $1 \mathrm{~cm}$ channel, b) at the $3 \mathrm{~cm}$ channel, c) $\Delta[\mathrm{CCO}]$ at the $1 \mathrm{~cm}$ and $3 \mathrm{~cm}$ channels, and d) [ $\left.\mathrm{tSO}_{2}\right]$ for $\mathrm{hNIRS}$ at the $1 \mathrm{~cm}$, and $3 \mathrm{~cm}$ channels and $\mathrm{mNIRS}$ device. Dashed lines indicate pacing episodes.

Figure 3.1. a, The probe for the hNIRS algorithm and b, the experimental setup of hNIRS at $10 \mathrm{~mm}, 30 \mathrm{~mm}$ and $40 \mathrm{~mm}$ for USB 4000, QE 65000, and Avantes.

Figure 4.1. Time course series of hNIRS- measured changes of TAVI patient during RVP are shown in a) [Hb] and [HBO2], b) Total $\mathrm{Hb}$ and [StO2], c) $\Delta[\mathrm{CCO}]$ and [StO2]. Ventricular pacing indicated in the dot-dashed boxes. The plot is shown for an example participant.

Figure 4.2. Changes in the cytochrome $C$ oxidase and total hemoglobin concentration during breath holding $(\mathrm{BH})$ measured by hNIRS at the optode separation of $4 \mathrm{~cm}$. The plot is shown for an example participant.

Figure 4.3 . Changes in the cytochrome $\mathrm{C}$ oxidase redox state measured by hNIRS using 8optimized wavelengths and mNIRS at the optode separation of $4 \mathrm{~cm}$. The plot is shown for an example participant. 


\section{LIST OF ABBREVIATIONS}

hNIRS.

Hyperspectral Near-infrared Spectroscopy

mNIRS Multispectral (multi wavelength) Near-infrared Spectroscopy

CW Continuous wave

EMR. Electromagnetic radiation

TPSF. Temporal point spread function

FD. Frequency domain

APD. Avalanche photodiode

TOI/TSI Tissue oxygen index/Tissue saturation index 2,3 BPG. 2,3 disphophoglycerate TCA. Tricarboxylic acid ATP. Adenosine triphosphate NADH. .Nicotinamide adenine dicucleotide

ETC Electron transport chain

MBLL Modified Beer-Lambert Law

DPF. Differential pathlength factor

TAVI. Transcatheter aortic valve insertion rCCO. Reduced state of Cytoschome C oxidase oCCO. Oxidized state of Cytochrome $\mathrm{C}$ oxidase redoxCCO Redox state of Cytochrome C oxidase 


\section{Chapter one}

\section{Introduction and background}

\section{1 - The physics of light transport in tissues}

Light is created from the electromagnetic radiation (EMR) of any wavelength which can give an effect on the skin depending on various degrees of absorption. The behavior of EMR depends on its wavelengths and represent a form of photon energy. The shorter the wavelength, the higher the energy penetrates into the tissue. Hence, the interaction of light and tissue are based on the propagation of light as well as its utilization in biological materials. In summary, there are two predetermined factors (the NIR light wavelength and the characteristic absorption spectra of the chromophores) and two variables (the concentration of the chromophores and the distance of the detected photon travel into the tissue) that affect the amount of absorption of the tissue.

The simplest form of light attenuation in a thin tissue layer is described by the Beer Lambert law. It states that the amount of light absorbance has a linear relationship with the specific absorption coefficient, concentration and the path length travel distance through the medium of the substance. The light intensity is equivalent to the depth of penetration where it is defined as the mean path length of the photons through the tissue. However, the interaction between light and tissue depends on constituents of tissue, optical properties of tissue and propagation where biological tissues is a highly scattering medium due to its inhomogeneity structure. As a result, the path length travel will increase based on the tissue region and wavelength of light. Therefore, for multiple scattering media such as tissue, the simple given from above cannot be applied. 
When light travels into the tissue, it will get absorbed as well as scattered in a forward direction. The angular probability of the incident photon travel along the initial direction $i$, being scattered into a different direction $s$ is given by the phase function $\mathrm{f}(i, s)$. The phase function is known as the function of the cosine of the scattering angle where, the mean cosine is expressed as the anisotropy factor of scattering, $\mathrm{g}[2,7]$. This scattering parameter is one of the optical properties which depends on the size and shape of the particle as well as the given wavelength of the incident light. The anisotropy factor $\mathrm{g}$ is defined as:

$$
\begin{gathered}
g=\int_{-1}^{1} \cos \theta f(\cos \theta) d \cos \theta \\
\text { if } g=\left\{\begin{array}{l}
-1: \text { complete backwark scattering } \\
0: \text { isotropic scattering } \\
1: \text { complete forward scattering }
\end{array}\right.
\end{gathered}
$$

Since biological tissues are known as strongly forward-scattering media, therefore the typical values of $\mathrm{g}$ are from 0.69 to 0.99 which correspond to an average scattering angle of 25 to 45 degrees. [3]. Tissue absorption is represented by the absorption coefficient $\left(\mu_{a}\right)$ which is defined as the amount of photon light that gets absorbed into the tissue per unit path length. On the other hand, the scattering coefficient denotes the scatter of light in biological tissue as the amount of the photon scattering in tissue per unit path length [4].

\section{2 - Near infrared spectroscopy in the human brain}

Near-infrared spectroscopy can be used as an index of the brain and living tissue oxygenation that has been used widely since 1977 when Jobsis demonstrated the potential methods of measuring oxygen saturation in the mammalian tissues. Since then, it has been widely used in research as 
well as in the clinical settings. It has been known to be a non-invasive real-time measurement for the cerebral region.

The propagation of Near-infrared light in tissue is based on the light transportation in tissue with the relative magnitudes of optical absorption and scattering. The principle of light propagation in the brain by NIRS can be shown in Figure 1.1. Under the reflectance optical property, NIRS light penetrates through the human head following a banana-shaped pathway where the depth of photon penetration depending on the source to the detector distance, then a small fraction of photons reaches to the detector. [5-6]. The typical distance of the separation distance on the human's head is no more than $4 \mathrm{~cm}$ due to the decreasing of light intensity lead to the limit of NIRS reading signals.
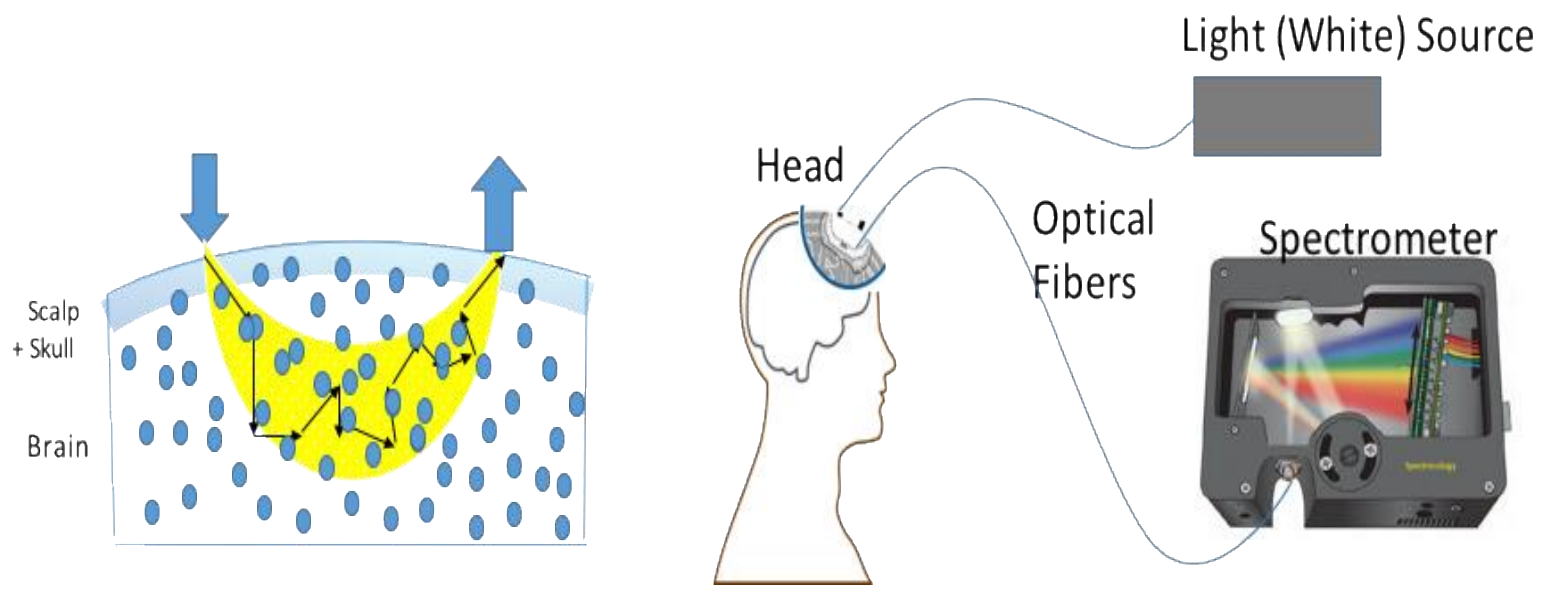

Figure 1.1 Principle of light propagation in the brain by NIRS.

The amount of light absorbance has a linear relationship with the specific absorption coefficient, concentration and the path length travel distance through the medium of the substance. The light intensity is equivalent to the depth of penetration where it is defined as the mean path length of the 
photons through the tissue. The relative transparency to light of biological tissue are mainly in the NIRS spectral window $(650-1000 \mathrm{~nm})$. This choice of wavelength is based on the absorption spectra of the major tissue chromophores such as water, deoxy, oxyhemoglobin and cytochrome c oxidase.

\section{3 - The history of Near Infrared Spectroscopy in the human brain}

The discovery of using near-infrared spectroscopy for monitoring brain oxygenation saturation was introduced since 1977 by Francis Jobsis. He worked at Duke University, North Carolina to show the ability to monitor, continuously and noninvasively the attenuation spectrum across the animal's head by not only on the near-infrared absorption bands of hemoglobin but also determine accurately the terminal member of the respiratory chain, cytochrome c oxidase [1]. Using this technique, Jobsis and his colleagues successfully demonstrated the changes in cerebral oxygenation in laboratory animals within the NIR range from $650 \mathrm{~nm}$ to $1100 \mathrm{~nm}$ ('optical window'). As the first, NIRS clinical studies on sick newborns and adult cerebrovascular patients, which were published in 1984 and 1985 by Delpy, Brazy and Ferrari [1,2]. The authors demonstrated the first quantitative measurement of various oxygenation and hemodynamic parameters. In his article, Jobsis [1] has described that the redox state of the copper band of cytochrome c oxidase, an enzyme that is responsible for about $90 \%$ of the cellular oxygen consumption could also be monitored. Since then, three major chromophores: oxyhemoglobin, deoxyhemoglobin and cytochrome c oxidase become the targets to most of the researchers and clinicians for cerebral studies. However, due to the amount of concentration of cytochrome C oxidase is less than $10 \%$ of haemoglobin concentration, it is difficult to achieve an accurate 
measurement for this potential intracellular marker of tissue oxygenation. At the present time, no commercial system included the cytochrome c oxidase measurement.

In 1989, the first commercial system built by Hamamatsu Photonics K.K (Hamamatsu City, Japan) that used the single-channel continuous wave (CW) NIRO-1000 was created for the clinical application. However, the first generation of medical NIRS spectroscopy system using a continuous wave approach to provide the concentration changes in deoxy and oxyhemoglobin was in 1992 by Somanetics (Troy, MI, USA) for a single - channel NIRS spectroscopy brain oximeter (INVOS 3100). The methodological development of near-infrared spectroscopy since then has made significant progress using different principles such as spatially resolved spectroscopy (SRS), phase modulation spectroscopy (PMS) and time-resolved spectroscopy (TPS). From 1980 to 1995, the development of NIRS spectroscopy prototypes expanded to a further nine companies, but there are only four of these companies are still producing NIRS product in 2011.

\section{4- Different NIRS spectroscopy measurements}

There are four main types of experimental techniques in the field of Near infrared spectroscopy: Continuous wave, time domain, frequency domain and spatially resolved spectrometers. Their principles, advantages and disadvantages of the individual method can be described by the following categories. Figure 1.2 has shown the schematic presentation of these spectrometers.

\subsection{1 - Time- resolved spectroscopy}

Time-resolved spectroscopy technique is also called the time-of-flight or time domain system. A short NIR light pulse of a few picoseconds was employed by a solid- state laser enter the medium to reflect the light intensity change measurement. This results in a different distribution of the total 
path length of a photon traveling that depends on the characteristic of the medium based on the amount of light that gets absorbed or scattered [8]. From the shape of this distribution and its temporal point spread function (TPSF), this technique has the ability to determine the absorption coefficient, the scattering coefficient and mean optical path length of tissue. Since the time domain spectrometer requires the stabilization of the instrument and the need for high-speed emitterdetection, it is the most expensive and technically complicated method which is not suitable for clinical monitoring [7].

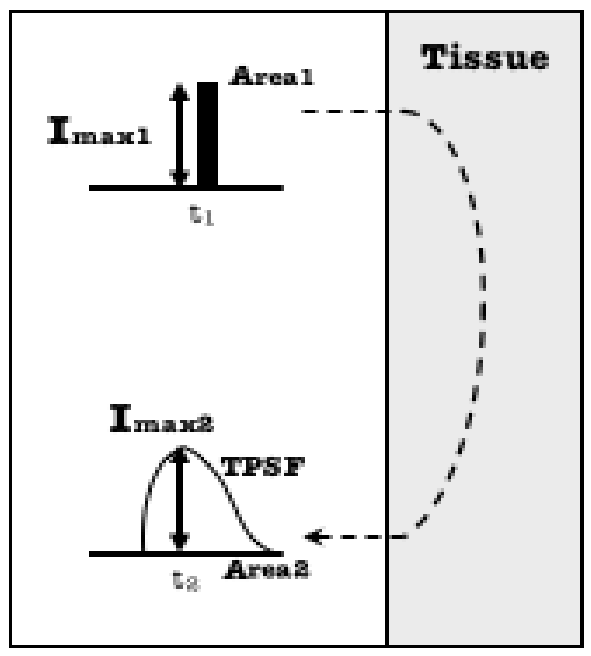

a) Time-domain NIRS

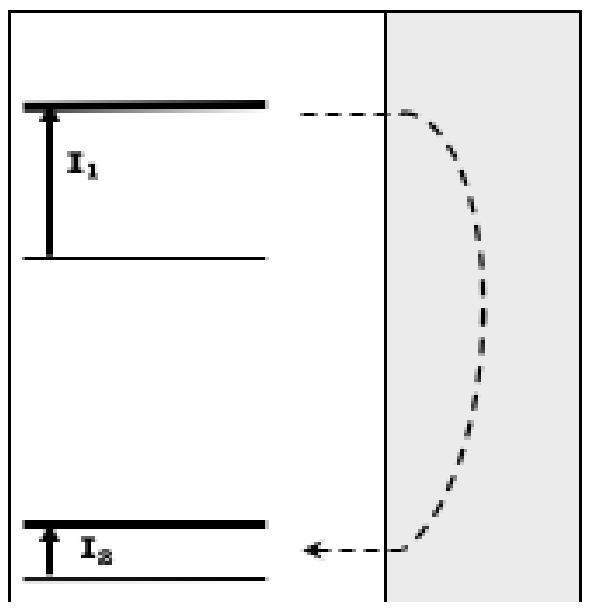

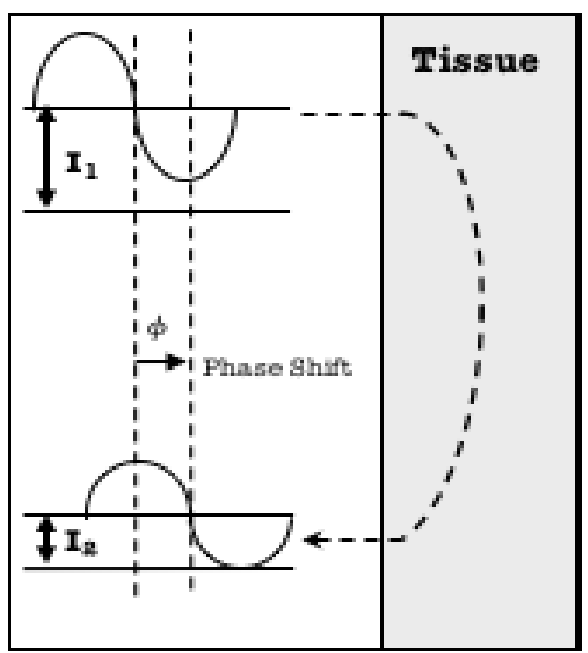

b) Frequency-domain NIRS

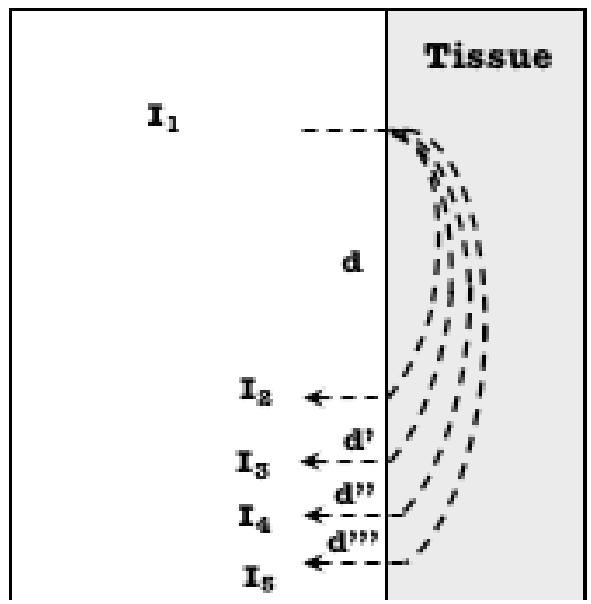

Figure 1.2 Different techniques used in the field of Near Infrared Spectroscopy: Time-domain, Frequency-domain, Continuous-wave and spatially-resolved NIRS. From Delpy \& Cope [11] 


\subsection{2 - Frequency domain spectroscopy}

In frequency domain or phase-modulated measurements, a NIR light source was employed to measure the intensity of light (DC component) as well as the phase shift and the modulation depth (AC component) when light passed through a tissue, in comparison with the initial intensity modulated input at radio frequency (more than $100 \mathrm{MHz}$ ). FD NIRS is capable of measuring the absorption coefficient of the tissue directly from the changes in the back-scattered signal's amplitude without using the information about photon path length. However, the linear relationship between the phase shift and path length factor does not hold the modulation frequencies higher than $200 \mathrm{MHz}$ which minimize the expansion of the number of channels [12]. Additionally, the phase noise and the penetration depth remain as the major limitations of FQ spectrometer. [13].

\subsection{3 - Continuous wave spectroscopy}

Continuous wave NIR spectroscopy is the earliest to be developed and the most common form to use due to its simplicity and cost-effectiveness. It can use a continuous filtered white light source, multiple discrete wavelengths source or a broad band of wavelength (hyperspectral) to measure the attenuation of light using either photomultiplier, photodiode or avalanche photodiode (APD) 
detector [11]. The NIR light source is emitted at a constant frequency and amplitude as it passes through a medium to reflect the changes of the substances' concentration through the modified Beer-Lambert Law or diffusion approximation theory. The CW NIRS show the trend in the changing values which only can yield the relative concentration changes of the substances or chromophores due to the lack of knowledge of photon path length. However, there are several possibilities to the quantity the changes in concentrations of the chromophores over the years.

\subsection{4 - Spatially resolved spectroscopy}

Spatially resolved spectroscopy (SRS) is one of the applications of CW NIRS to monitor the concentrations of the chromophores. It is the combination of several light sources and detectors in a single probe to create a multiple closely spaced source-detector distances to measure light attenuation as the function of source-detector separation. Hence the knowledge of photon path length is not a requirement to make the calculation the change and the absolute measurement of hemoglobin. The tissue oxygen index (TOI) or tissue saturation index (TSI) is defined as the amount of oxygenated hemoglobin over the total amount of hemoglobin can be calculated based on the slop of light attenuation versus the detector distance from the initial light source by using photon diffusion theory [7]. TOI has the significant correlation with the cerebral oxygen saturation to become a useful tool to identify the threshold for cerebral ischemia $[7,8]$.

Furthermore, SRS approach using the diffusion approximation model has a high degree of sensitivity and specificity to the changes of the intracranial and extracranial [14]. It helps to extract the superficial layer of brain tissue to measure the concentration of the chromophore in the brain without the inference of the intracerebral tissue on the light attenuation. 


\section{5 - Tissue Chromophore}

\subsection{1 - Oxyhemoglobin and deoxyhemoglobin as oxygen transport agents}

Hemoglobin is a complex ion-molecule called metalloprotein, which can be found in red blood cells (RBCs). Its major function is to carry $97 \%$ of oxygen in the blood, and the rest is dissolved in the plasma membrane.

It consists of a quaternary structure which creates four polypeptide chains: 2 alpha and 2 beta chains. Each of these polypeptide chains contains a heme group with a centered ion molecule to bind with oxygen. The haemoglobin can bind up to 4 oxygen molecules as so-called oxygenated haemoglobin while deoxygenated haemoglobin is the deoxygenated form of haemoglobin which is not bound to any oxygen molecules.

Haemoglobin has an important role in carrying the oxygen inhaled by the lungs to the tissues (oxygenated haemoglobin) and delivering the carbon dioxide from the tissues back to the lungs (Deoxygenated haemoglobin) [15]. As the results, oxygenated haemoglobin $\left(\mathrm{HbO}_{2}\right)$ has the bright red color due to oxygen molecules in the arterial blood region while deoxygenated haemoglobin $(\mathrm{Hb})$ is carried in the venous blood flow which has the darker red.

The binding between oxygen and haemoglobin molecule at the four heme groups cause the shape and the conformation of the haemoglobin to change, although the binding does not happen simultaneously. When the first heme group binds with oxygen, there is a small change in the structure of haemoglobin to stimulate the binding to the rest of the oxygen molecules. There are various factors such as partial pressure of oxygen, temperature, $\mathrm{pH}, \mathrm{CO} 2$, the metabolite 2,3 disphophoglycerate (2,3-DPG) can affect the binding of oxygen to haemoglobin [11]. For typical haemoglobin in an adult, the $\mathrm{pH}$ level is at 7.4 , at $27^{\circ} \mathrm{C}$ and $3.578 \mathrm{kPa}[7,11]$, haemoglobin transfers 
into a tense form which oxygen affinity is low and tends to release oxygen in the tissue when haemoglobin has lower partial pressure, $\mathrm{pH}$, but higher in $\mathrm{CO} 2$ and 2,3BPG. Inversely, haemoglobin can exit into a relaxed form where it wants to bind with oxygen molecules due to the higher in oxygen affinity, oxygen partial pressures, $\mathrm{pH}$, but lower in $\mathrm{CO}_{2}$ and 2,3 BPG. The level of oxygen saturation $\left(\mathrm{SO}_{2}\right)$ as the fraction of oxygenated haemoglobin relative to the total haemoglobin in the blood represents the percentage of hemoglobin binding to oxygen can be calculated based on the following formula:

$$
\begin{array}{r}
\mathrm{SO}_{2}=\frac{\mathrm{HbO}_{2}}{\mathrm{HbO}_{2}+\mathrm{Hb}}(\%) \\
\text { where, } t \mathrm{Hb}=\mathrm{HbO}_{2}+\mathrm{Hb}
\end{array}
$$

Haemoglobin molecules are responsible for the absorption of light for RBCs, but depending upon to the degree of oxygenation. Figure 1.3 shows the NIRS absorption spectra of these compounds. The absorption spectra of $\mathrm{Hb}$ ranges from $650 \mathrm{~nm}-1000 \mathrm{~nm}$ while $\mathrm{HbO}_{2}$ shows a broad peak from 700- 1150nm. The intersection between $\mathrm{Hb}$ and $\mathrm{HbO}_{2}$ is called the isosbestic point where they have the same molar absorptivity $(810 \mathrm{~nm})$ and helps to measure the concentration of total haemoglobin [16]. 


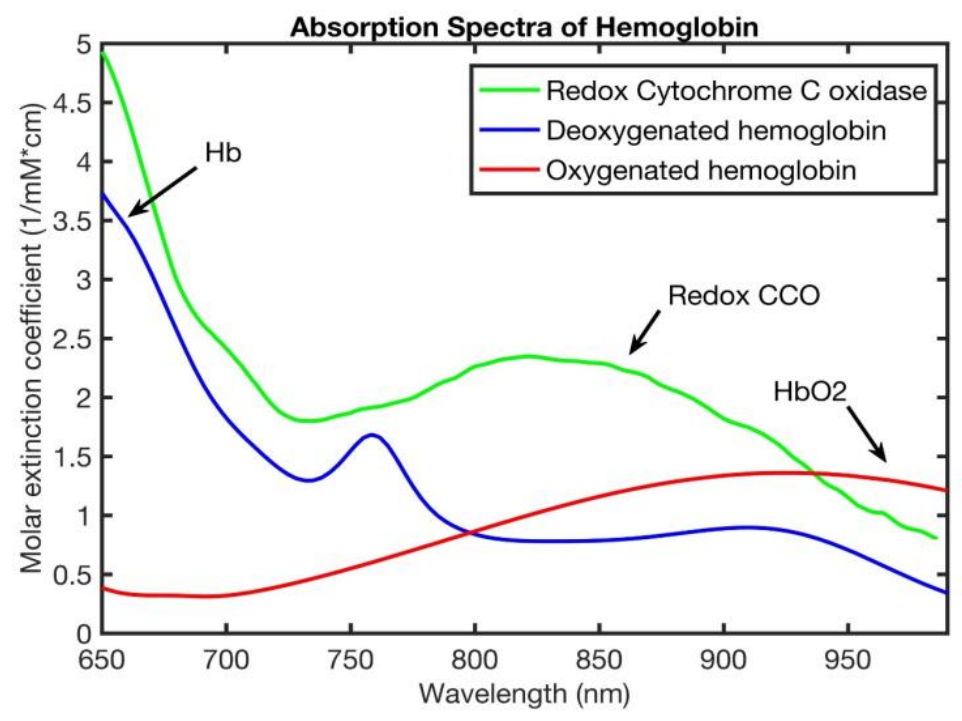

Figure 1.3. The absorption spectra of oxyhemoglobin, deoxyhemoglobin and cytochrome $c$ oxidase.

\subsection{2 - Cytochrome c oxidase as an oxygen-consume agent}

The intention of measuring cytochrome C oxidase since 1977 by Franz Jobsis has denoted the significant role of this chromophore in the tissue metabolism study. Cytochrome C Oxidase (CCO) is known as the last enzyme in the respiratory electron transport chain, which plays an important part in the aerobic metabolism of glucose to generate energy. In order to understand the NIRS signal of $\mathrm{CCO}$, the basic knowledge of the biochemical processes by the aerobic metabolism are needed to apprehend.

The metabolic pathway which is called glycolysis, results in the breakdown of glucose into pyruvate then at the citric acid cycle or the tricarboxylic acid (TCA) cycle, it is converted to AcetylCoA in the mitochondria. During TCA cycle, ATP (adenosine triphosphate) and NADH (Nicotinamide adenine dinucleotide) are produced, which NADH is fed into the oxidative phosphorylation (electron transport) pathway. The oxidative phosphorylation pathway is the process that the redox reactions to generate ATP from the electron donors to electron acceptors interactions are carried out by a series of protein complexes embedded in the inner membrane of 
mitochondria. These protein complexes are known as complexes I to $\mathrm{V}$, where the electron transport train (ETC) from the complex I to IV account for the oxidation of NADH whereas the production of ATP is created at complex V. Figure 1.4 shows the graph of cellular metabolism with electron transport chain and $\mathrm{CCO}$ interaction [17].

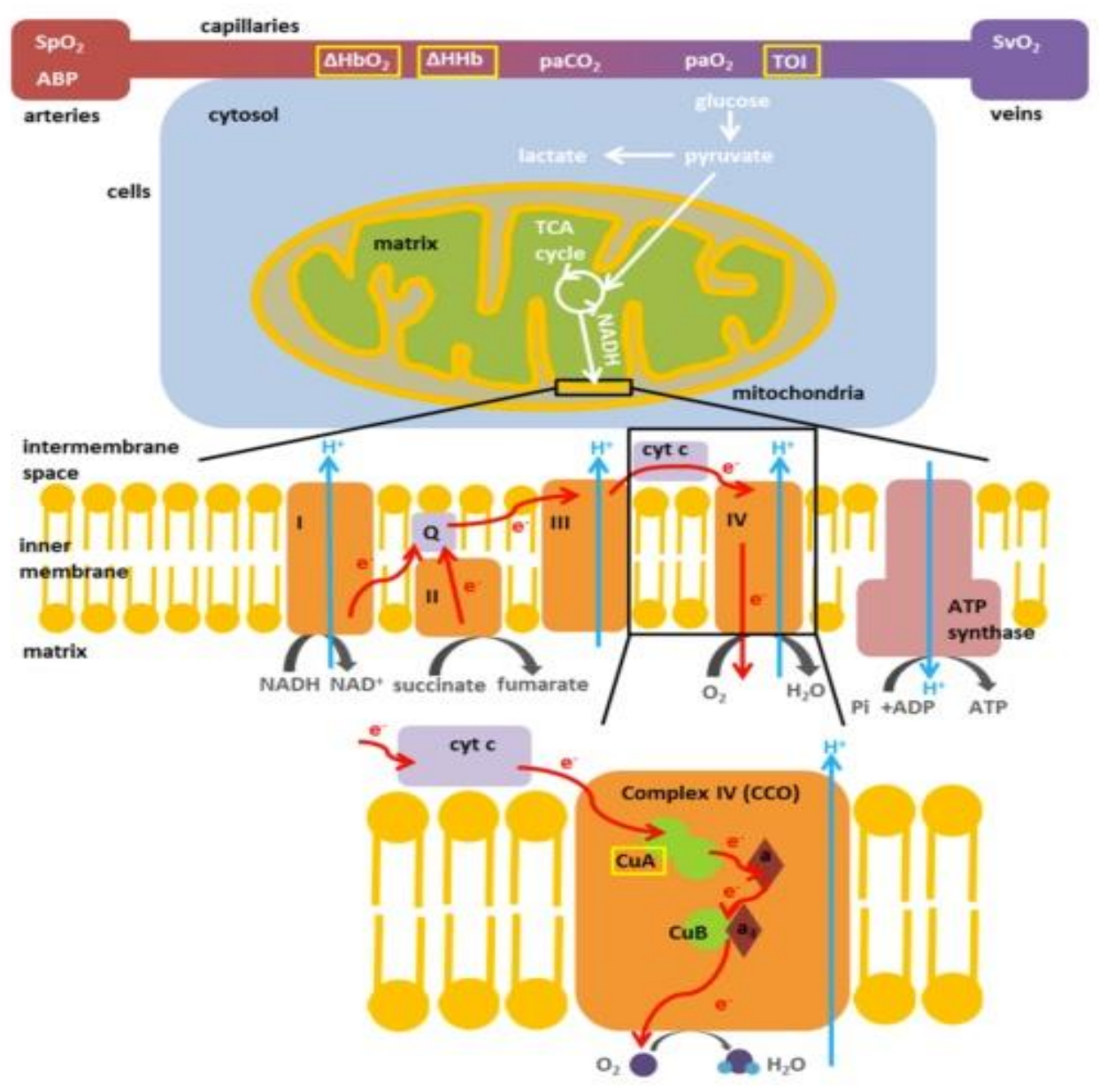

Figure 1.4. The schematic graph of cellular metabolism with electron transport chain and CCO interaction. From Bale et al [17].

NADH acts the electron donors which its electrons will release a large amount of energy due to oxidation. Complex I accepted electrons from NADH and transferred to a lipid soluble called 
ubiquinone $(\mathrm{Q})$ to produce ubiquinol $(\mathrm{QH} 2)$. Complex II is the parallel electron transport pathway to complex I by delivering additional electrons into Q. QH2 is oxidized by complex III to reduce to the highly-water soluble electron transport protein called cytochrome c. Complex IV is also known as cytochrome $\mathrm{C}$ oxidase $(\mathrm{CCO})$ received the delivered electrons from cytochrome $\mathrm{c}$ and transferred to the molecular oxygen to produce water. During these transportations of electrons in complex I, III and IV, there is a proton electrochemical gradient across the inner mitochondrial membrane is produced which drives the synthesis of ATP via ATP synthase. This is known as complex V in the ETC.

CCO often gets considered as the final protein complex in the ETC, which it has a highly complicated structure. The structure of $\mathrm{CCO}$ contains 4 redox active metal centers: $\mathrm{CuA}$, haeme $\mathrm{a}, \mathrm{CuB}$ and heme $\mathrm{a} 3$ where they follow the simple linear electron pathway transportation to oxygen in CCO by transferring four electrons from each of four cytochrome c molecules to bind with two oxygen molecules of water at a binuclear center. The binuclear center is the site of oxygen reduction to create water where haem $\mathrm{a} 3$ and $\mathrm{CuB}$ centres form. The $\mathrm{CuA}$ and haeme donate electrons to this binuclear unit. As a result, $\mathrm{CuA}$ centers is not involved in oxygen reaction. Although, all of the components of this enzyme has associated with optical transitions, the $\mathrm{CuA}$ center dominates a strong peak of the absorption spectrum in the NIR range of $830 \mathrm{~nm}$ due to the presence of oxygen intermediates while the remain components are in the visible range [18].

The CuA copper centers is a simple one- electron acceptor which consists of a unique $\mathrm{Cu}-\mathrm{Cu}$ dimer that gives a distinct absorbance spectrum by the NIRS signal. The rates of electron transfer at this copper centers correspond to the changes in its the redox state. Cooper et al have demonstrated specifically the significance of $\mathrm{CuA}$ redox-state change in vivo as well as the relationship between oxygen and the $\mathrm{CuA}$ redox state which ultimately is followed by the CCO redox state (oCCO). 
Therefore, oCCO is dependent on the rate of electrons transfer in the ETC as well as the rate of oxygen in the cells. There are a variety of factors that affect the redox state of CCO in vivo such as the presence of inhibitor, $\mathrm{pH}$, oxygen tension, ATP turn-over, the proton electrochemical gradient or even the substrates in the ETC system.

The changes in redox state of CCO can be monitored by using the difference spectrum between the oxidized and reduced states where the UCL group has shown that by measuring the difference spectrum the changes in oxidized $\mathrm{CCO}$ or reduced $\mathrm{CCO}$ can be resolved. Gemma Bale et al have shown the proof of this statement by presenting two scenarios using the modified Beer Lambert law. The absorption spectra of CCO can be shown in Figure 1.5 based on the data from the UCL group.

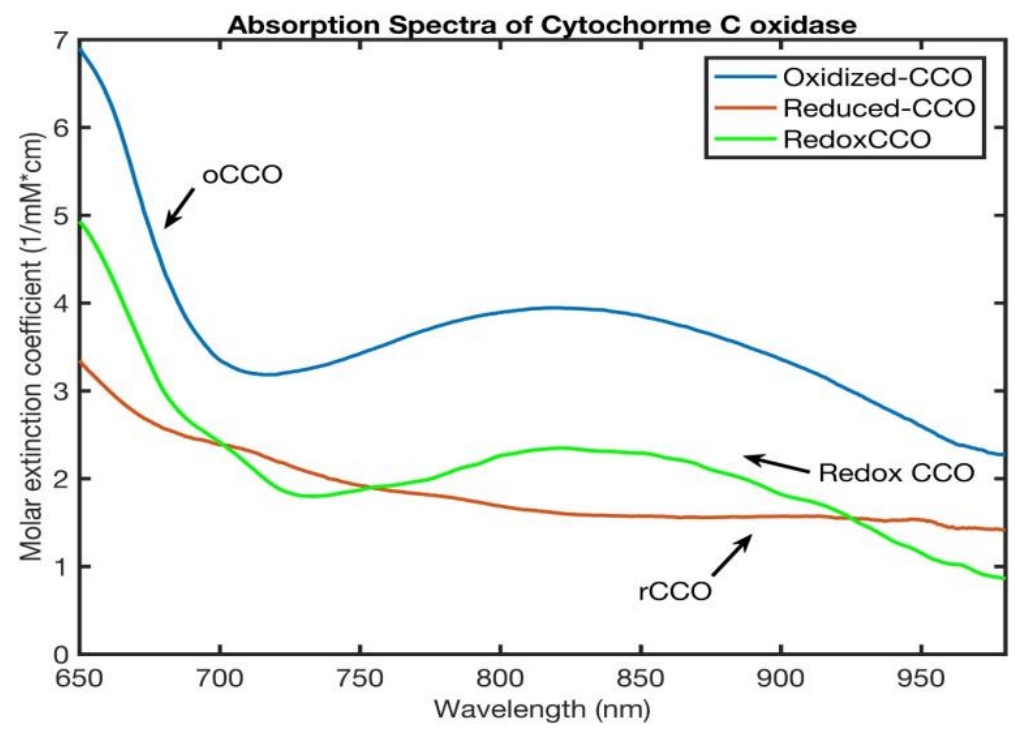

Figure 1.5. The absorption spectra of cytochrome c oxidase.

CCO has 3 different conformational states: fully oxidized (oCCO), partially oxidized (poCCO) and reduced (rCCO), where each state has a different affinity. The fully reduced state of $\mathrm{CCO}$ is considered as the resting state of CCO since the chromophore has enough transferred electrons to 
become fully reduced. The fully oxidized which is known as the first scenario shows the highest activity when heme $\mathrm{a} 3$ and $\mathrm{CuB}$ nuclear unit are fully oxidized at the initial time to to a later time t1. According to the Beer-Lambert law, the attenuation of $\mathrm{CCO}$ at the $\mathrm{t}_{\mathrm{o}}$ and $\mathrm{t}_{1}$ can be defined as the product $\mathrm{CCO}$ concentration $(\mathrm{C})$, its molar extinction coefficient $(\varepsilon)$, and the optical path length of light (1) as below:

$$
\begin{aligned}
& A\left(t_{o}\right)=l \varepsilon_{o C C O} C_{o C C O}\left(t_{o}\right) \\
& A\left(t_{1}\right)=l \varepsilon_{o C C O} C_{o C C O}\left(t_{1}\right)+l \varepsilon_{r C C O} C_{r C C O}\left(t_{1}\right)
\end{aligned}
$$

In a short time period of time, the total concentration of CCO is constant and does not change, hence the attenuation of the fully oxidize CCO at the initial time to is the same as the attenuation at time $\mathrm{t}_{1}$ when $\mathrm{CCO}$ is at the second conformational state which is partially oxidized and reduced. Alternately, the equation (1) and (2) can be written as:

$$
C_{o C C O}\left(t_{o}\right)=C_{o C C O}\left(t_{1}\right)+C_{r C C O}\left(t_{1}\right)
$$

Since the changes in the redox state of CCO concentration can be obtained by using the difference spectrum between oCCO and $\mathrm{rCCO}$, the difference extinction coefficient spectrum can be described as $\varepsilon_{\text {redoxcco }}$ or $\varepsilon_{\text {diff }}=\varepsilon_{\text {occo }}-\varepsilon_{\text {rCco }}$. The changes in the attenuation of redox CCO can be expressed as:

$$
\begin{aligned}
& \Delta A=A\left(t_{1}\right)-A\left(t_{0}\right)=l\left(\varepsilon_{o C C O}-\varepsilon_{\text {rCCO }}\right) \Delta C_{o C C O} \\
& \Delta A=l \varepsilon_{\text {redoxCCO }} \Delta_{o C C O}
\end{aligned}
$$


Similarly, the equation (3) and (4) can be estimated from the second scenario, where the initial attenuation at the time to for $\mathrm{CCO}$ exists in both oxidized and reduced forms for this case is equal to the attenuation at a later time which also exists in both forms:

$$
C_{o C C O}\left(t_{o}\right)+C_{r C C O}\left(t_{o}\right)=C_{o C C O}\left(t_{1}\right)+C_{r C C O}\left(t_{1}\right)
$$

It has been shown that $\mathrm{CCO}$ has the higher specificity in the brain compared to the hemoglobin chromophore [19]. Even though CCO is the sufficient enzyme in the living tissue, its concentration is considerably less than hemoglobin at least by an order of magnitude, the measurement of CCO in the brain for the NIRS techniques is substantially challenging. There has been a significant amount of work on measuring cytochrome in the brain from the previous studies [19-21]; however, the standard measurement for this is unknown.

\section{6 - The clinical need for cerebral oxygenation measurement during cardiac arrest}

Out-of-hospital cardiac arrest (CA) occurs in over 350,000 people in North America and has a low survival rate of $<10 \%$. More importantly, the rate of survival with good neurologic function is only $8 \%$; ischemic brain injury due to hypoperfusion is a major cause of neurological injury where no flow or low flow states prevent the transport of oxygen to the brain [22]. The American Heart Association has acknowledged that brain injury after cardiac arrest should be a critical focus in

clinical research [23]. Cerebral monitoring is a crucial technology to provide optimal care to prevent ischemic brain injury after cardiac arrest.

Unlike Pulse oximeter, which it considers only the arterial saturation of hemoglobin and also depends on pulsatile flow, NIRS not only can provide a local of assessment of oxygenation in all 
vascular components in the instant reading even at low blood flow such as cardiac arrest, but also utilizes more wavelengths to characterize different chromophores in the cerebral monitoring.

There are a variety of commercially cerebral oximeters which are used based on the principle of near-infrared spectroscopy for the cerebral measurements. Table 1.1 [24] shows that there are different applications have used to monitor the cerebral measurement by using a few wavelengths during cardiac arrest. It can be shown that there is a significant amount of patients in these studies.

Table 1.1. Near infrared spectroscopy monitoring during cardiac arrest.

\begin{tabular}{lllll}
\hline Study & $\begin{array}{l}\text { Number of } \\
\text { Patients }\end{array}$ & $\begin{array}{l}\text { Type of } \\
\text { oximeters }\end{array}$ & $\begin{array}{l}\text { Location of } \\
\text { cardiac } \\
\text { arrest (CA) }\end{array}$ & Outcome \\
\hline Ahn 2013 & 50 & $\begin{array}{l}\text { Equanox } \\
7600\end{array}$ & $\begin{array}{l}\text { In of } \\
\text { hospital CA } \\
\text { and Out of } \\
\text { hospital CA }\end{array}$ & $\begin{array}{l}\text { Return of spontaneous } \\
\text { circulation }\end{array}$ \\
\hline Asim 2014 & 23 & $\begin{array}{l}\text { INVOS } \\
5100 \mathrm{c}\end{array}$ & $\begin{array}{l}\text { Out of } \\
\text { hospital CA }\end{array}$ & $\begin{array}{l}\text { Return of spontaneous } \\
\text { circulation }\end{array}$ \\
\hline $\begin{array}{l}\text { Fukuda } \\
2014\end{array}$ & 69 & $\begin{array}{l}\text { INVOS } \\
5100 \mathrm{c}\end{array}$ & $\begin{array}{l}\text { Out of } \\
\text { hospital CA }\end{array}$ & $\begin{array}{l}\text { Return of spontaneous } \\
\text { circulation }\end{array}$ \\
\hline Nishiyama & 1772 & INVOS & $\begin{array}{l}\text { Out of } \\
\text { hospital CA }\end{array}$ & $\begin{array}{l}\text { Return of spontaneous } \\
\text { circulation, survival, } \\
\text { neurologic outcome }\end{array}$ \\
\hline 5100c & & Equanox & $\begin{array}{l}\text { In of } \\
\text { hospital CA }\end{array}$ & $\begin{array}{l}\text { Return of spontaneous } \\
\text { circulation }\end{array}$ \\
\hline Parnia 2014 & 34 & $\begin{array}{l}7600 \\
\text { and INVOS }\end{array}$ & & \\
\hline
\end{tabular}

This new technology has the ability to guide the quality of cardiopulmonary resuscitation (CPR) and as a tool for individual prognostication such as the return of spontaneous circulation (ROSC) and nerologic outcomes. Cournoyer et al have shown that patients experienced ROSC, surviving to discharge, and with good neurologic outcome after cardiac arrest have the higher values of NIRS 
oxygen saturation compared to their counterparts [24]. Notably, it suggests that researchers and clinicians could use this technology during CA for the evaluation of ROSC prognosis.

\section{7 - Basic anatomy of the human head}

The structure of the human head is complex which has the function to protect the brain organ in the body. The brain is protected by multiple layers: the scalp, skull, various membranes (meninges) and the cerebrospinal fluid (CSF) which can be shown from the Figure 1.6. It starts from the scalp and the skull or cranium as the first layer to shield the brain from the physics impacts. The next layer is the meninges which consist of three different membranes: the dura, arachnoid and pia mater. The dura mater is the most superficial and thickest membrane which is composed of dense irregular connective tissue. [25]. The middle meningeal membrane layer is the very thin, delicate arachnoid mater. This layer surrounds loosely the last meningeal membrane layer called pia matter which it is bound very tightly to the surface of the brain. The space between the arachnoid mater and the pia mater is filled with CSF and the blood vessels that serve the brain. [26]. The brain is composed of the gray matter, and the white matter where the gray matter or the cerebral cortex is on the outer surface of the cerebrum which contains numerous types of neurons and the white matter is the inner next to the gray matter. It comprises mainly of axons and dendrites to connect with neurons from the gray matter. [2]. 

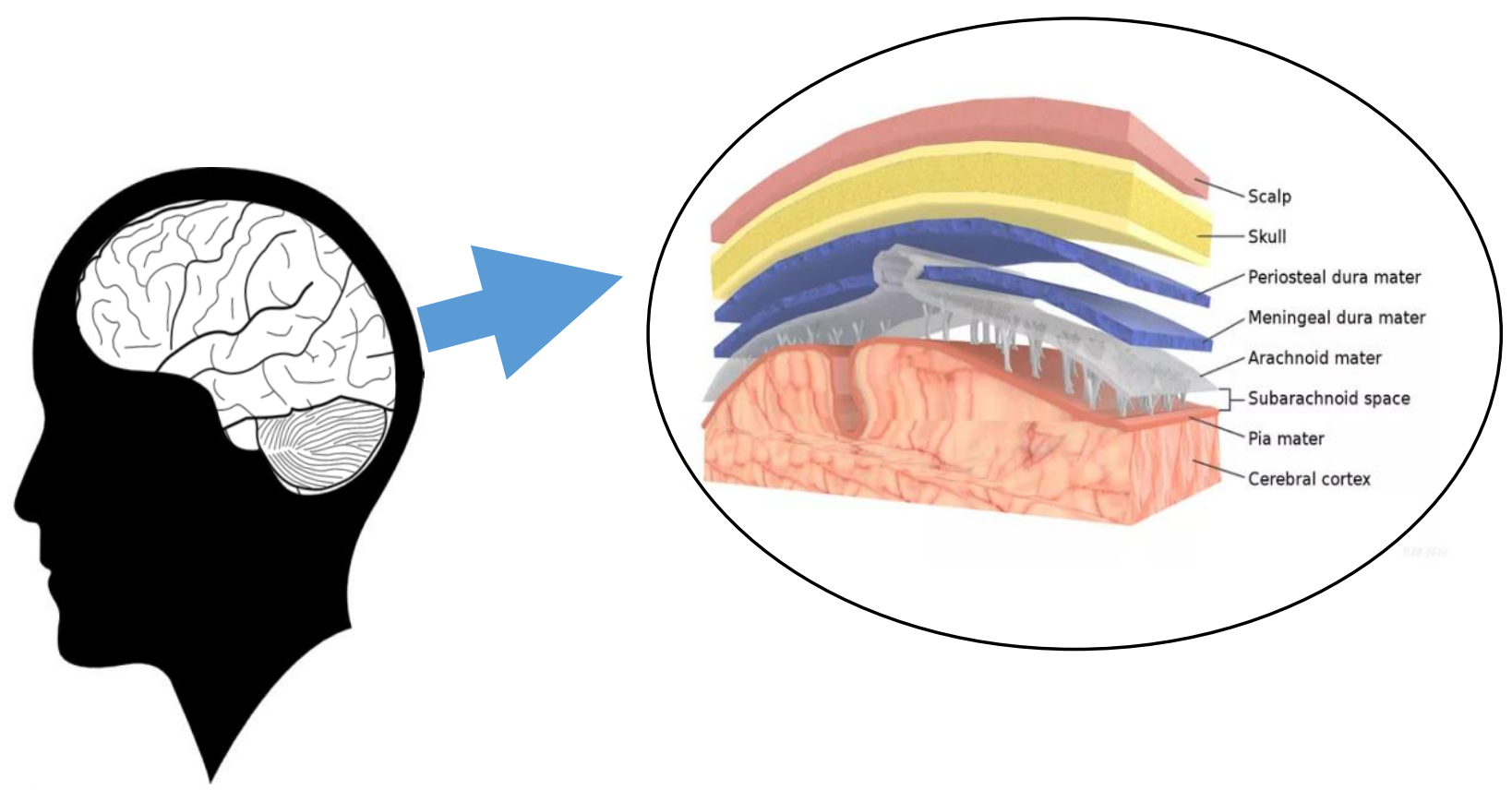

Figure 1.6. The basic anatomy of the human head. [27].

\section{8 - NIRS and current two- layer model of the human head}

Before the light passes through the brain, it must first pass through all the different superficial tissue layers as described from the previous section. In some of these layers, it might contain chromophores which can affect the measurement of the cerebral oxygenation by a variable amount. Previous studies documented that the values of NIRS measurement of the interested layer (brain) indeed gets the interference from all layer above it [28-30]. It is important to differentiate the contribution of extracerebral tissue to consider the influence of them to the behavior of light. Hemoglobin is known as the most dominant chromophore within the near infrared window where only the scalp and brain are the two experiencing blood flow with time-varying optical properties [31]. The remaining layers are assumed to be constant, and homogeneous and the scattering changes can be ignored in relation to the magnitude changes of absorption [32]. The scalp and 
skull in human adults are approximately $5-7 \mathrm{~mm}$ and $7-8 \mathrm{~mm}$ thick, respectively which imposes a challenge to measure cerebral oxygen saturation in the brain since it depends on the optical properties of these layers as well as the thickness with respect to the source and detector distance. Under these conditions, the 2-layer model can be adopted to describe the optically active properties of the brain. Figure 1.7 shows the schematic representation of NIRS light to the brain using the 2layer model. Here, the top layer contains the skull, scalp CSF and the various surrounding membrane layers which is called the extracranial layer whereas, the bottom layer contains the outer regions of the brain, namely as the intracranial. The 2-layer model can approach by using a homogeneous model for the top layer and semi-infinite medium as the bottom layer incorporating with either diffusion theory or a modified Beer-Lambert law. The use of time domain and frequency domain spectrometer systems tend to provide sufficient information regarding the measurement of oxygen saturation in the bottom layer [33-35]. However, the lack of stabilization and the cost from these systems could be challenging for the head NIRS measurements.
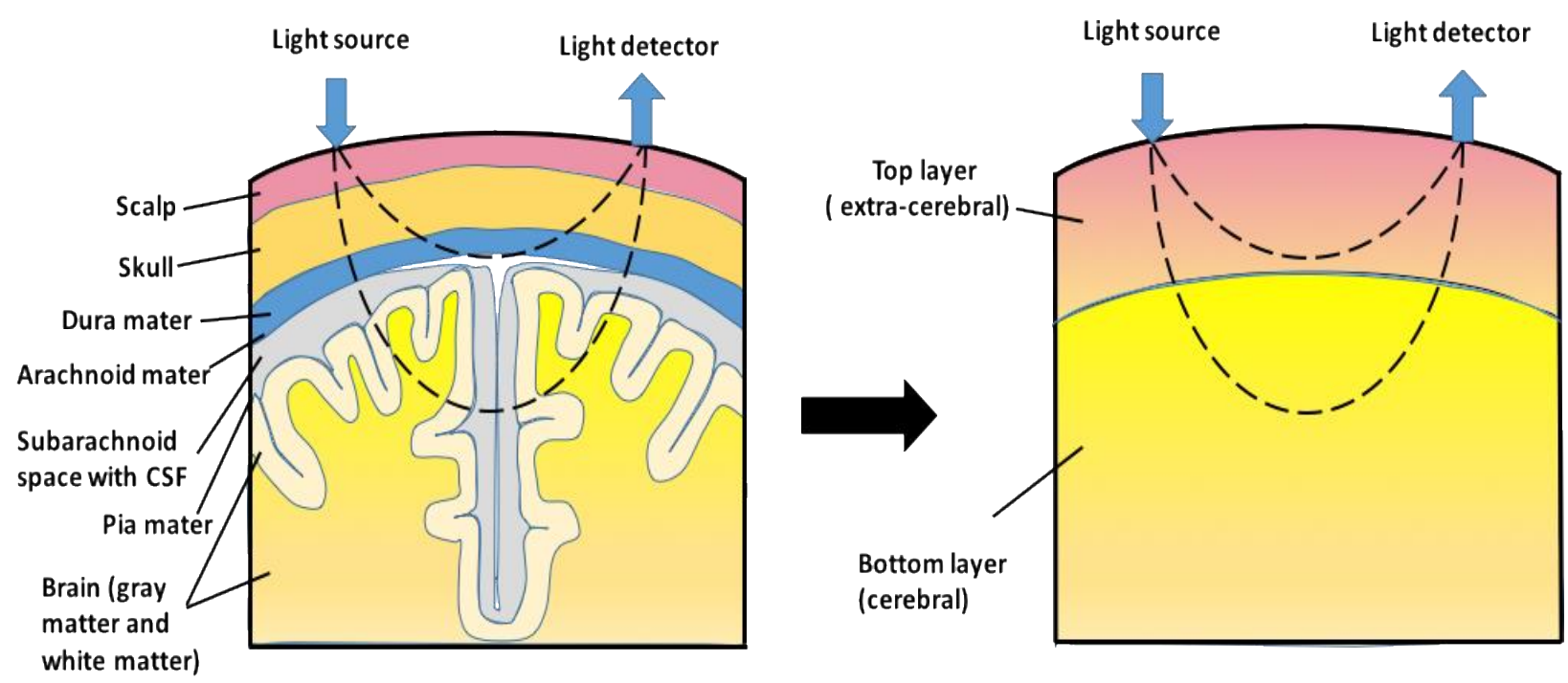

Figure 1.7. Schematic presentation of the brain in NIRS. 


\section{9 - Algorithms}

\subsubsection{Modified Beer- Lambert Law on the commercial multispectral NIRS}

As it was mentioned before, when light travels into a biological tissue medium, photons not only absorb but scatter into different directions, which depend on the optical properties of that medium. Hence, the travel path length of photons becomes longer than the distance from the source to the detector. The modified Lambert-Beer Law (MBLL) which is known as the basic of continuouswave near-infrared tissue spectroscopy (cwNIRS) can be used to determine the observance of the light [36-38] in this case:

$$
O D=\log \left(\frac{I_{o}(\lambda)}{I(\lambda)}\right)=\varepsilon(\lambda) C d D P F(\lambda)+G=\mu_{a} d D P F+G
$$

where OD is the absorbance or optical density (OD), $\mathrm{I}_{\mathrm{o}}$ is the incident intensity $\left(\mathrm{W} / \mathrm{cm}^{2}\right), \mathrm{I}$ is the detected intensity $\left(\mathrm{W} / \mathrm{cm}^{2}\right), \varepsilon$ is the extinction coefficient of the chromophores with respect to the wavelength $\left(\mathrm{OD} * \mathrm{~cm}^{-1 *} \mu \mathrm{M}^{-1}\right), \mathrm{C}$ is the concentration of the chromophore $(\mu \mathrm{M}), \mathrm{d}$ is the separation distance from the source to the detector $(\mathrm{cm}), \mathrm{DPF}$ is the differential pathlength factor (unit-less), which is equal to the ratio of the total mean path length of detected photons to the distance of the source-detector [39]. G is known as the background noise or a scattering term (OD), which depends on optical geometry and characteristic of tissue [40]. The absolute values of concentration of chromophores by using this approach cannot be used in the application for biological tissues due to the uncertainty values of DPF, G, and a number of different chromophores. The DPF values can be used from the literatures for the given types of tissue. The measurements of the DPF values cannot be done in the continuous-wave system; instead, they can be determined by using the time- 
resolved system to accurately estimate these values [41-42]. The DPF values are dependent on the source-detector distances, but they do not depend on the wavelengths in the near infrared range which is necessary to obtain useful information. Most of the previous studies assume that $\mathrm{G}$ is constant during the measurement period, which is true in a homogeneous medium with a constant scattering property [43]. One of the studies explained that to able to eliminate the influences of $\mathrm{G}$, two different distances of source-detector can be used with the condition that the distance between each one and the light source is large enough that the emitted light received from each source can penetrate across the detected tissue [40]. Hence, the chromophore concentration changes from the measured attenuation changes can be obtained:

$$
\Delta O D_{1}=\sum_{i} \varepsilon_{i} \Delta[C]_{i} D P F d
$$

In most applications of optical brain imaging, the changes in $\mathrm{HbO}_{2}$ and $\mathrm{Hb}$ concentrations are considered. Therefore, the MBLL equation for a particular wavelength through 2 different sourcedetector distances $r_{1}$ and $r_{2}$ can be presented as a set of the simultaneous equation as below: [40]

$$
\sigma O D=O D_{2}-O D_{1}=\left(\varepsilon_{\mathrm{HbO}_{2}} C_{\mathrm{HbO}_{2}}+\varepsilon_{\mathrm{Hb}} C_{\mathrm{Hb}}\right) D P F\left(r_{2}-r_{1}\right)
$$

Since $\mathrm{C}_{\mathrm{HbO}}, \mathrm{C}_{\mathrm{Hb}}$ are the concentration of oxy hemoglobin and deoxy hemoglobin which are two unknown parameters, there should be at least 2 different wavelengths needed to be used to calculate the changes of optical density by knowing the extinction coefficients of these two wavelengths $\lambda_{1}$ and $\lambda_{2}$. Hence the changes in the concentration of $\mathrm{Hb}$ and $\mathrm{HbO}_{2}$ can be solved through the system of the linear equation based on equation (1.10) from above $[36,44,45]$ : 


$$
\begin{aligned}
& \Delta[H b]=\frac{\varepsilon_{H b O_{2}}^{\lambda_{2}} \frac{\Delta O D^{\lambda_{1}}}{D P F_{1}^{\lambda_{1}}}-\varepsilon_{H b O_{2}}^{\lambda_{1}} \frac{\Delta O D^{\lambda_{2}}}{D P F_{2}^{\lambda_{2}}}}{\left(\varepsilon_{H b}^{\lambda_{1}} \varepsilon_{H b O_{2}}^{\lambda_{2}}-\varepsilon_{H b}^{\lambda_{2}} \varepsilon_{H b O_{2}}^{\lambda_{1}}\right) d} \\
& \Delta\left[H b O_{2}\right]=\frac{\varepsilon_{H b}^{\varepsilon_{1}} \frac{\Delta O D^{\lambda} 2}{D P F^{\lambda}}-\varepsilon_{H b}^{\lambda_{2}} \frac{\Delta O D^{\lambda_{1}}}{D P F^{\lambda}}}{\left(\varepsilon_{H b}^{{ }_{1} \varepsilon_{H b O_{2}}^{\lambda}}-\varepsilon_{H b}^{\varepsilon_{2}} \varepsilon_{H b O_{2}}^{\lambda_{1}}\right) d}
\end{aligned}
$$

Furthermore, the cerebral tissue oxygen saturation can also be obtained:

$$
t S O_{2}=\frac{\varepsilon_{H b}^{\lambda_{1}}-\varepsilon_{H b}^{\lambda_{2}} \frac{\sigma O D^{\lambda_{1}}}{\sigma O D^{\lambda_{2}}}}{\frac{\sigma O D^{\lambda_{1}}}{\sigma O D^{\lambda_{2}}}\left(\varepsilon_{H b O_{2}}^{\lambda_{2}}-\varepsilon_{H b}^{\lambda_{2}}\right)-\left(\varepsilon_{H b O_{2}}^{\lambda_{1}}-\varepsilon_{H b}^{\lambda_{1}}\right)}
$$

The accuracy of the calculation can be improved by using a multi-linear regression model to fit for each chromophore spectrum [11] as the number of wavelengths exceeds the numbers of the given chromophores, which can be expressed as the matrix as below:

$$
\left[\begin{array}{c}
\Delta H b O_{2} \\
\Delta H b
\end{array}\right]=\left[\begin{array}{cc}
\varepsilon_{H b O_{2}}^{\lambda_{1}} & \varepsilon_{H b}^{\lambda_{1}} \\
\varepsilon_{H b O_{2}}^{\lambda_{2}} & \varepsilon_{H b}^{\lambda_{2}}
\end{array}\right]^{-1} \cdot\left[\begin{array}{c}
\Delta O D^{\lambda_{1}} \\
\Delta O D^{\lambda_{2}}
\end{array}\right] \cdot d D P F
$$

where are the extinction coefficients for oxy and deoxy hemoglobin at wavelength 1 and 2 . If there is additional chromophore need to be resolved, changes in the concentration must be measured at a minimum of three wavelengths. Most of the systems currently available commercially use the maximum of four wavelengths based on the continuous wave theory of the MBLL [46]. 


\subsubsection{1 - The availability of multiple source-detector distances on the commercial multispectral NIRS}

As mention from the previous, the cwNIRS cannot directly measure the DPF like time domain and phase domain NIRS techniques based on the MBLL equation where DPF depends on the distance between the source- detector. Hence, this optode distance is known as one of the significant factors affect sampling regions where the maximum signal intensity and the signal-to-noise ratio will change accordingly. Therefore, it is essential to use the appropriate combination of the source to detector distances to maximize the sensitivity to cerebral oxygenation measurement from the extracerebral and cerebral regions as well as improve spatial resolution for mapping cortical activation [47]. There are different NIRS devices commercially available for measuring cerebral oxygen saturation under the principle of spatially resolved technology or 2-layer model, which are FDA- approved such as INVOS 5100 (Covidien Boulder, CO), FORE-SIGHT (CAS Medical Systems Inc, Brandford, CT) and Equanox 7600 (Nonin Medical Inc, Plymougth, USA). By using the MBLL method, they can detect the changes in cerebral saturation and eliminate the contribution of superficial tissue by using more than one source -detector distance. However, despite the similar system using near- infrared wavelength to detect the changes in the concentration of $\mathrm{Hb}$ and $\mathrm{HbO}_{2}$, there are several technical differences between these devices. This can be shown by the Table 1.2 [48]. 
Table 1.2. Applications of cerebral measurements using mNIRS.

\begin{tabular}{|c|c|c|c|}
\hline rSO2 sensor & $\begin{array}{l}\text { Nonin } \\
\text { (Equanox 7600) }\end{array}$ & $\begin{array}{l}\text { Covidien } \\
\text { INVOS }\end{array}$ & $\begin{array}{l}\text { CASMED } \\
\text { FORE-SIGHT }\end{array}$ \\
\hline Wavelength & $\begin{array}{l}3 \\
730 \mathrm{~nm}, 810 \mathrm{~nm} \text {, } \\
\text { and } 880 \mathrm{~nm}\end{array}$ & $\begin{array}{l}2 \\
730 \mathrm{~nm} \text { and } 810 \mathrm{~nm}\end{array}$ & $\begin{array}{l}4 \\
690 \mathrm{~nm}, 780 \mathrm{~nm}, \\
804 \mathrm{~nm}, \text { and } 850 \mathrm{~nm}\end{array}$ \\
\hline Source & 2 & 1 & 1 \\
\hline Light paths & 4 & 2 & $1-2$ \\
\hline $\begin{array}{l}\text { Short detector/ } \\
\text { long detector }\end{array}$ & $20 / 40 \mathrm{~mm}$ & $30 / 40 \mathrm{~mm}$ & $15 / 50 \mathrm{~mm}$ \\
\hline
\end{tabular}

While INVOS features only 2 wavelengths, Equanox 7600 features 3 wavelengths, FORE-SIGHT features 4 wavelengths of near-infrared light. In addition, the distances between the various light emitters and detectors are different. The source-detector distances are $20 \mathrm{~mm}$ and $40 \mathrm{~mm}$ for the Equanox classic 7600, 30mm and 40mm for the INVOS 5100 and for the FORE-SIGHT the distances are $15 \mathrm{~mm}$ and $50 \mathrm{~mm}$. However, some previous studies have shown that extracranial contamination appears to significantly affect the cerebral measurement and the $\mathrm{RSTO}_{2}$ measurement from all three devices had pair-wise differences in either mean absolute values, repeatability and/or dynamic analysis [5, 49-52].

\subsubsection{2 - Limitation of the modified Beer-Lambert Law algorithm}

The MBLL follows a structure as a linear relation which is assumed that the changes in concentration and absorbance over a brief short interval, then the effect of the change in scattering is added. This only works for very small sections as the error will increase when the section 
becomes large [53]. Alternatively, when the change in concentration grows large in the MBLL's law may not reflect this change in the measured value correctly.

Besides that, the MBLL are based on two assumptions when estimating the attenuation with more than two wavelengths: (1) the absorption of the tissue changes homogenously and (2) the scattering loss is constant $[46,54,55]$. The first assumption does not hold for most of the practical cases since the changes of the tissue absorption are usually inhomogeneous. This focuses on the investigation of the cross-talk effect between all the absorbing substances since for any individual value at a time point is influenced by the change in the concentration of other chromophores [54]. Some previous studies have shown that the combinations of the optimal wavelength for the dual wavelengths can minimize these errors $[41,55]$. Furthermore, the second assumption might not be qualified in cerebral oxygenation saturation measurement. Besides the tissue scattering changes when neuronal activation occurs in the brain, the assessment of the cross-talk of scattering changes into the estimated changes in chromophore concentrations has not been reported previously [43, 56,57] .Besides, most of the commercial devices applying this MBLL algorithm tend to have a lack of consistency regarding measurements of oxygenation saturation by using a constant mean path length independent of the wavelength which the wavelength dependence is ignored. Furthermore, oxCCO is known as the most abundant enzymes in biological tissues; however, it is one of the most notable challenges for these devices to calculate the changes in oxCCO.

\subsection{2 - Diffusion approximation}

The transportation of photon in biological tissue can be modeled analytically by the radiative transfer equation (RTE), which is considered equivalent to the numerical Monte Carlo method 
without any computational time limitation. The deviation of RTE (or the Boltzmann equation) is based from the principle of conservation of energy, where coherence, polarization, and nonlinearity are ignored [4], which can be obtained as the equation from below:

$$
\frac{\partial L(\vec{r}, \hat{s}, t) / c}{\partial t}=-\hat{s} . \nabla L(\vec{r}, \hat{s}, t)-\mu_{t} L(\vec{r}, \hat{s}, t)+\mu_{s} \int_{4 \pi} L\left(\vec{r}, \hat{s}^{\prime}, t\right) P\left(\hat{s}^{\prime} \cdot \hat{s}\right) d \Omega^{\prime}+S(\vec{r}, \hat{s}, t)
$$

Where $L(\vec{r}, \hat{s}, t)$ is known as the spectral radiance with $\mathrm{r}$ denotes the position, $\mathrm{s}$ denotes the unit direction vector, t denotes time. $S(\vec{r}, \hat{s}, t)$ is a source in the volume element within the solid angle element per unit time. $P\left(\hat{s}^{\prime} . \hat{s}\right)$ is the phase function which only depends on the angle between the incident and scatters directions. It represents the amount of light propagating into the biological tissue at a direction $\hat{s}^{\prime}$ and being scattered into all direction to create a solid angle element $d \Omega$ around direction $\hat{s}^{\prime} . c$ is the speed of light inside the biological tissue and $\mathrm{u}_{\mathrm{t}}$ is the sum of the absorption coefficient and scattering coefficient. All the optical properties: the refractive index n, the absorption coefficient $\mu_{a}$, the scattering coefficient $\mu_{s}$, and the scattering anisotropy $g$ are assumed to be time-invariant but space variant. However, RTE equation is complicated to solve since it has six different variables $(x, y, z$, from $\vec{r}$, polar angle $\theta$, azimuthal angle $\phi$ from $\hat{s}$, and $t)$. It often gets simplified in the diffusion approximation with the assumption that the radiance in a predominantly scattering medium:

$$
\frac{\partial \Phi(\vec{r}, t)}{c \partial t}+\mu_{a} \Phi(\vec{r}, t)-\nabla[D \nabla \Phi(\vec{r}, t)]=S((\vec{r}, t)
$$

With $D=\frac{1}{3\left(\mu_{a}+\mu_{s}^{\prime}\right)}$ is the diffusion coefficient and $\mu_{s}^{\prime}=(1-g) \mu_{s}$ is the reduced scattering coefficient whereas the scattering coefficient describes as the reciprocal of the mean free path. Due 
to the anisotropy of the scattering, $\mu^{\prime}{ }_{s}$ is introduced to determine a series of random step sizes of $1 / \mu^{\prime}$ in the biological tissue.

Similar to most of the noninvasive medical applications method, the light source, and the detector are placed upon the surface of the tissue. It is not appropriate for the infinite- medium boundary for this geometry as well as for the diffusion theory due to the strongly anisotropic intensity of photon near the surface. Hence, a uniform, homogeneous semi-infinite medium can be solved the problem with given optical properties and extrapolated boundary correction as explained in detail by [58]. An analytical solution for a semi-infinite homogeneous medium for the continuous wave photon flux between source and detector regarding the absorption coefficient and the reduced scattering coefficient of the medium is given by the equation below:

$$
(\quad)=\frac{2}{(4)^{2}} \frac{S}{D} \frac{\exp \frac{a}{D} \div}{3} 1+\frac{a}{D} \div\left(z_{0}+z_{b}\right) \quad z_{b}+3 D 1 \frac{\left(z_{0}+z_{b}\right)^{2}+3 z_{b}^{2}}{2} 3+\frac{{ }^{2} \frac{a}{D}}{1+\frac{a}{D} \div}
$$

Where $\rho$ is the source-detector distance in the radial coordinate, $\mathrm{z}_{\mathrm{o}}=3 \mathrm{D}$, and $\mathrm{zb}_{\mathrm{b}}=2 \mathrm{D}$ are the boundary approximation values [59]. The absorption coefficient of the biological tissue can be model as:

$$
\mu_{a}(\lambda)=\varepsilon(\lambda)_{\mathrm{HbO}_{2}} C_{\mathrm{HbO}_{2}}+\varepsilon(\lambda)_{\mathrm{Hb}} C_{\mathrm{Hb}}+\varepsilon(\lambda)_{o C C O} C_{\mathrm{oCCO}}
$$

The reduced scattering coefficient is determined based on the simplified Mie theory which acts as the functions of wavelengths can be described by a power law:

$$
\mu_{s}^{\prime}(\lambda)=\mu_{s 800}{ }^{\prime}\left(\frac{\lambda}{800}\right)^{-\alpha}
$$


Where $\mu_{s 800}$ ' is the scattering prefactor which presents the reduced scattering coefficient at the isobetic point $(\lambda=800 \mathrm{~nm})$. The fixed value for the scattering power $\alpha$ can be found by Yeganeh et al [60].

\subsubsection{1 - Hyperspectral near-infrared spectroscopy algorithm using 2-layer model}

Most applications of NIRS use the diffusion approximation theory to exam the propagation of light in the brain. However, the drawback of many studies which have assumed that the optical properties of the diffused medium were homogenous. This is not true as the complex structure of the human head with different layers such as skin, skull, dura mater, etc. Some previous studies have attempted to use the frequency domain, and time domain NIRS approaches to separate the extracerebral layer and the cerebral layer. These methods are sufficient but highly cost in equipment and a relatively low signal to noise ratios are the significant drawbacks [61]. It is not possible to use one single wavelength with cwNIRS to separate the absorption and the scattering properties of the biological tissue, a different approach is using a broadband of wavelength or hyperspectral cwNIRS for the two-layer model can be used to improve the accuracy of the measurements of $\mathrm{Hb}, \mathrm{HBO}_{2}$, and $\mathrm{CCO}$ parameters. More than 1000 wavelengths from $650 \mathrm{~nm}$ to $1100 \mathrm{~nm}$ with the spectral resolution of $0.5 \mathrm{~nm}$ are utilized to determine not only changes in the concentration of $\mathrm{Hb}$ and $\mathrm{HbO}_{2}$ but also change in concentration of an important enzyme in the mitochondrial electron transport chain is cytochrome c oxidase. In this approach, given the complexity of the head, a simple modification for 2 -layer model is created where the top layer is corresponding to the extracerebral layer (skin, skull, scalp, cerebrospinal fluid) and the bottom layer reflects the cerebral layer (gray and white matter of the brain). This model depends on six 
different parameters: the source-detector distance, the absorption and reduced scattering of the top and bottom layer, and the top-layer thickness. The analytical solution of the light diffusion equation for two-layer geometry is modeled the head as a semi-infinite two-layer medium with two steps for the fitting procedure: (1) to determine the optical properties of the top layer using homogeneous model, and (2) with the determined values of optical properties of the top layer, the two-layer model is applied to determine the optical properties of the bottom layer. The changes in the concentration of the cerebral region for $\mathrm{Hb}, \mathrm{HbO}_{2}$, and $\mathrm{CCO}$ can be calculated by performing a non-linear least square fitting between the changes in fluence and absorbance:

$$
\ln \frac{\psi_{\lambda}\left(\mu_{a}+\Delta \mu_{a}, \mu_{s}{ }^{\prime}\right)}{\psi_{\lambda}\left(\mu_{a}, \mu_{s}{ }^{\prime}\right)} \stackrel{\text { fitting }}{\longrightarrow} \quad \ln \left(\frac{I(\lambda)}{I_{0}(\lambda)}\right)
$$

This hNIRS 2-layer model was validated by the previous studies on phantom and pig models of cardiac arrest and has shown significant correlations with other invasive measurements. [61-62]. Figure 1.8 shows the signal processing algorithm of the hNIRS two-layer model to assess the changes in concentrations of the chromophores in the cerebral region.
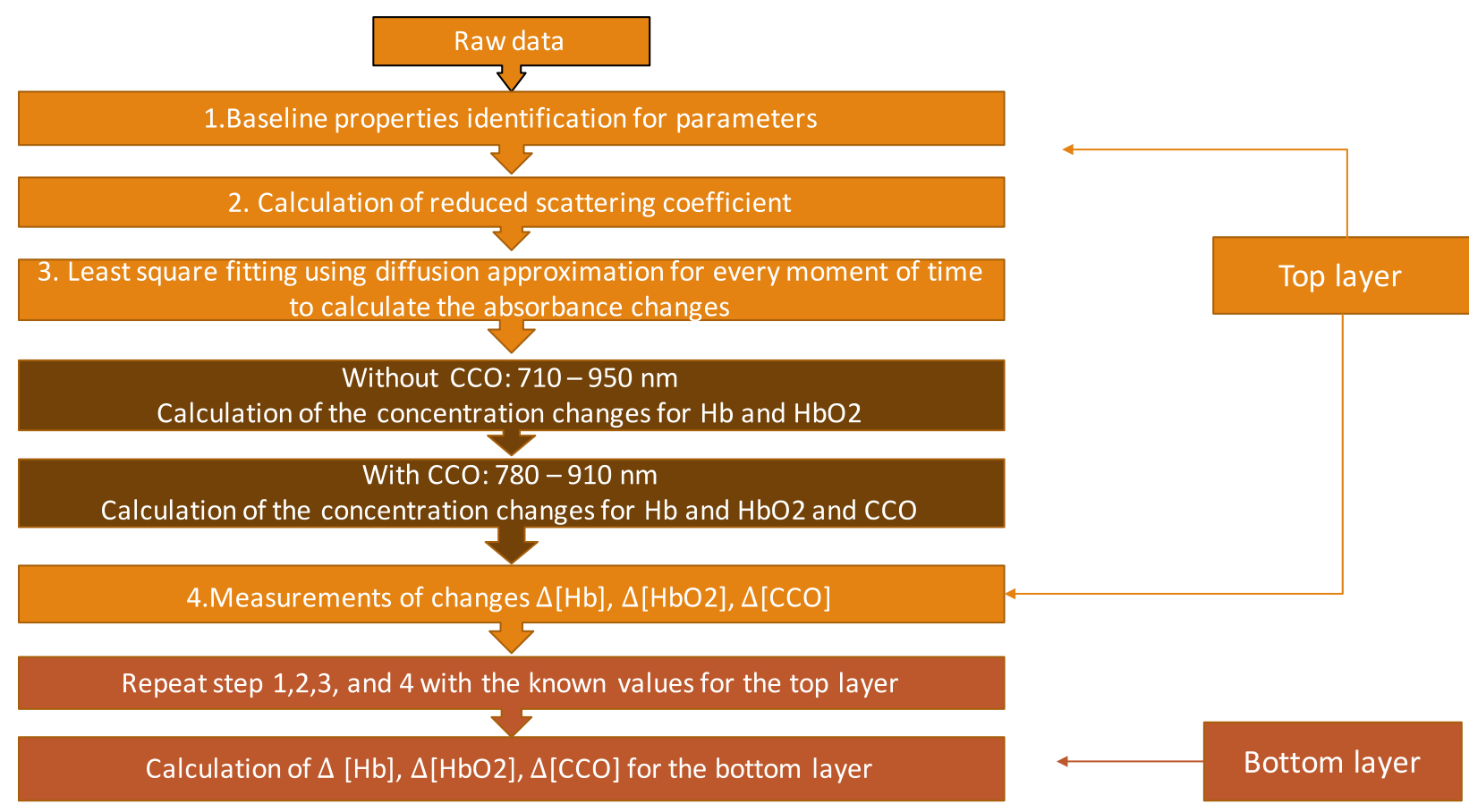
Figure 1.8. Hyperspectral NIRS data processing algorithm for changes in the concentrations of chromophores.

\subsection{0 - Wavelength selection for hNIRS algorithm}

Broadband spectroscopy offers a full range of measurement of wavelengths. However, it can be challenging to incorporate into wireless and multichannel systems for cardiac arrest procedures. Moreover, with a large number of wavelengths, the size of the computational problem also increase which leads to the larger memory requirement. Using an optimized set of wavelengths can be sufficient in term of computational time and provide critical results based on the continuous wave measurements. A recent study has employed a genetic algorithm (GA) to search for different combinations of wavelengths regarding determine the changes in the concentration of $\mathrm{HB}, \mathrm{HbO}_{2}$ and CCO accurately [20]. To validate the results, they were compared with the current gold standard of a broadband NIRS system across from 780nm to $900 \mathrm{~nm}$ at the spatial resolution of 1nm [63]. The gold standard plot can be seen in Figure 1.9. Matcher at el performed the comparison of several multi-wavelength NIRS algorithms to assess the separation of the CCO signal from the hemoglobin signals from different sets of data on human forearm muscle, adult rat head, and newborn piglet head [32]. The results showed that the higher number of wavelengths produced accurate simulations and improved the in vivo measurement. Relatively, D Arifler et al [20] found that the optical three wavelength combination only gives reasonable results for the changes in the hemoglobin signals but not for CCO. 


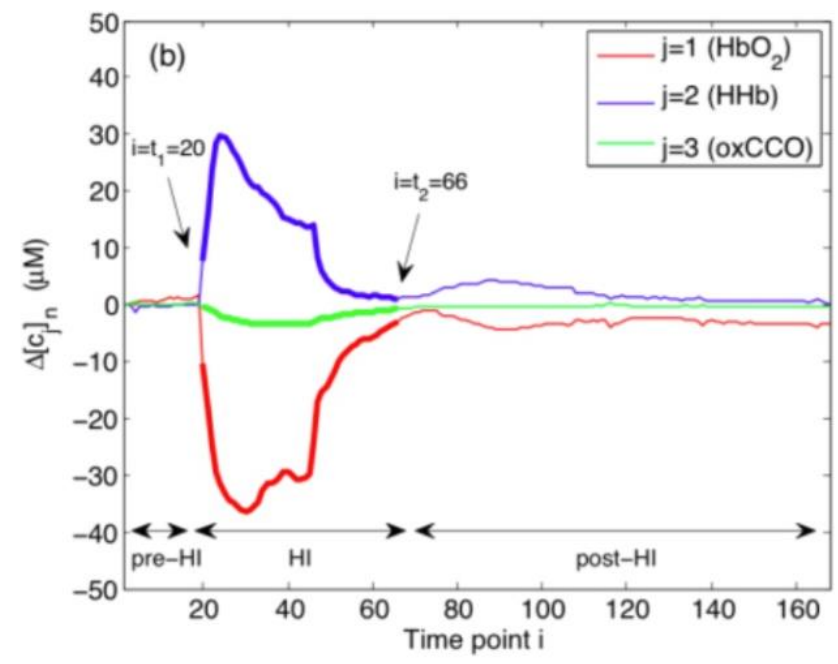

Figure 1.9. The gold standard values of the changes in concentrations of $\mathrm{HbO}_{2}, \mathrm{Hb}$, and $\mathrm{CCO}$ corresponding with $j=1,2$, and 3 , respectively.

When the combination number of wavelength increases; increasing from 3 to 4 , 5 , and 8 wavelengths, the errors against the gold standard result reduces. The recommended optimal wavelength combination for simultaneous assessment of three chromophores $\mathrm{Hb}, \mathrm{HbO}_{2}$, and $\mathrm{CCO}$ was found to have eight wavelengths: $784,800,818,835,851,868,881$, and $894 \mathrm{~nm}$; which need to be span across the range of the spectrum in order to resolve the shape of the CCO and separate it from the hemoglobin without having weak signal problems and achieve a solution with minimal crosstalk between chromophores. Hence, it is possible to reduce the number of measured wavelength without losing the high performance in estimating changes in cerebral chromophores concentrations.

\subsection{1 - Thesis organization and objectives}


The goal of this research to examine the feasibility of using hNIRS to detect changes in cerebral oxygenation and metabolism in cardiac arrest from the patients undergoing transcatheter aortic valve insertion (TAVI). Patients undergoing TAVI experience brief, controlled changes in cardiac output with hemodynamics similar to cardiac arrest this approach is based on the two-layer model of the human head where the top layer is corresponding to the extracerebral layer (skin, skull, scalp, cerebrospinal fluid) and the bottom layer reflects the cerebral layer (gray and white matter of the brain). It is hypothesized that NIRS can measure changes in the cytochrome Coxidase redox reflecting the changes in the cerebral metabolic rate of oxygen in TAVI and cardiac arrest with the longer distance between the light source and detector to give significant results of cerebral measurement. In addition, the measurement in estimating changes in cytochrome c oxidase concentration can be achieved with evaluated multiple wavelengths based on our NIRS algorithm at the certain optode seperations.

In this thesis, the method consisted of a few steps: First, to obtain the absolute measurement of the baseline optical properties in the brain of patients undergoing transaortic valve replacement (TAVI), a model for cardiac arrest. This can be performed by a spectral differential fit two-layer model analysis of the absorbance data in the near infrared range of 730 to $950 \mathrm{~nm}$. Once the optical properties of the baseline have accomplished, the measurement of changes in concentrations of chromophores during the cardiac arrest was performed. In order to improve the accuracy of the two-layer model as well as the hNIRS algorithm, we applied the same principles for the breath holding subjects with different optode separations to differentiate the extracranial contamination. As an extra constraint and validation of the results, we compared the data measurements of NONIN (mNIRS) from the MBLL model with our hNIRS model. Furthermore, we modified the Matlab software package of the broadband spectra using the semi-infinite homogeneous medium for the 
diffusion equation to the given selective wavelength combinations of NIRS from the prior literature reviews based on the animal model to compare the results based on the data processing algorithm for multispectral NIRS as Figure 1.10.
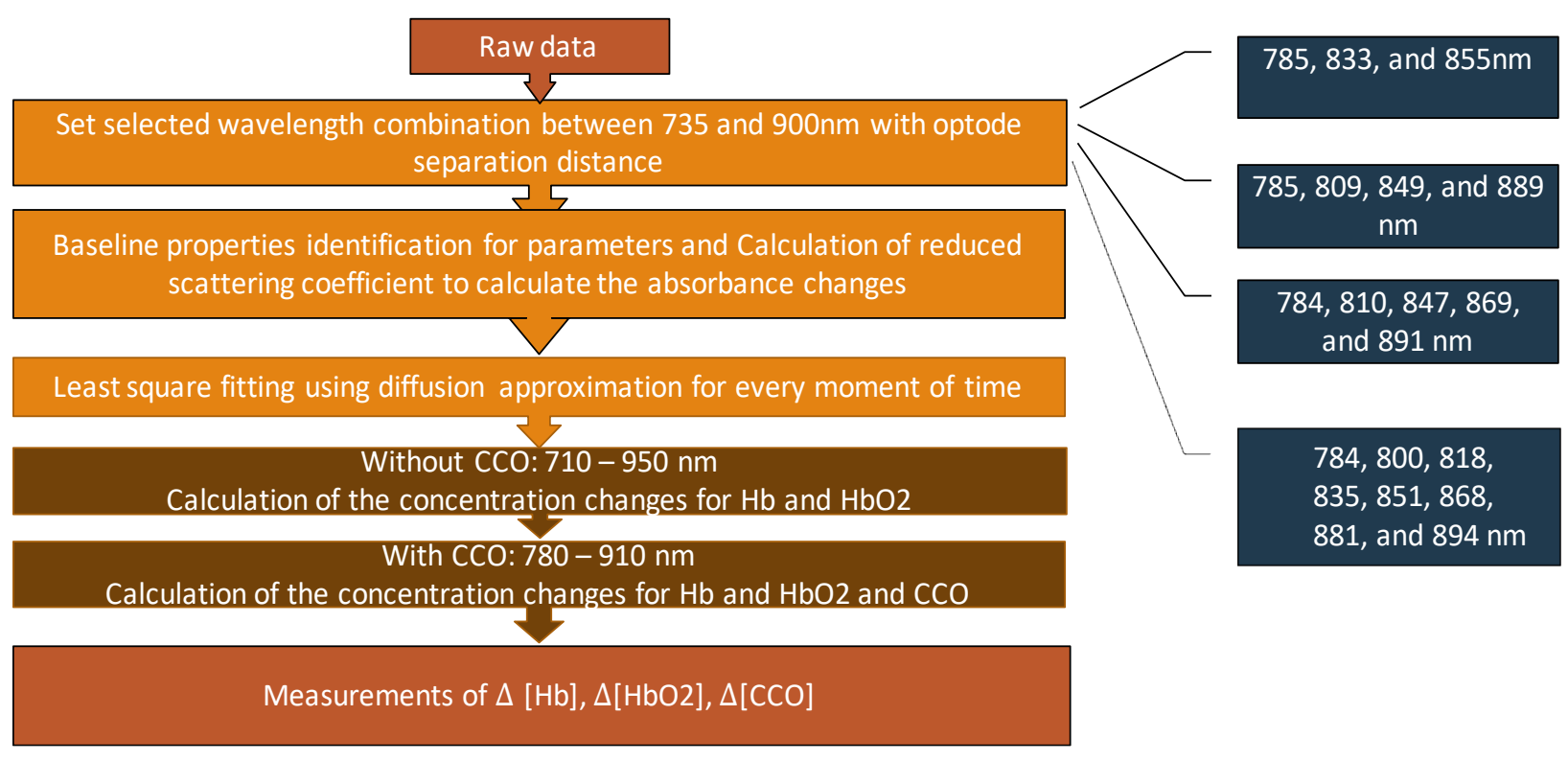

Figure 1.10. Multispectral NIRS data processing algorithm for changes in the concentrations of chromophores.

Chapter 2 presents a submitted journal manuscript in the Journal of Biomedical Optics on the assessment of hNIRS in the brain during cardiac arrest. Chapter 3 presents the additional methodology as well as the results of breath holding hNIRS measurements on different sourcedetector spacing distances at 10,30 and $40 \mathrm{~mm}$. It also shows the results and discussion of the 2layer model and breath holding measurements. In addition, Chapter $\mathbf{4}$ is the final chapter which presents conclusions and future work. 
Chapter 2

Journal manuscript 


\title{
Hyperspectral near infrared spectroscopy assessment of the brain during hypoperfusion
}

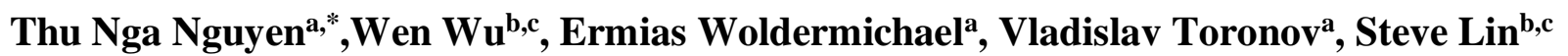

${ }^{a}$ Ryerson University, Faculty of Science, Department of Physics, 350 Victoria Street, Toronto, ON, Canada, M5B

$2 \mathrm{~K} 3$

${ }^{\mathrm{b}}$ Li Ka Shing Knowledge Institute, St. Michael's Hospital, 30 Bond Street, Toronto, ON, Canada, M5B 1W8

${ }^{\mathrm{c}}$ University of Toronto, Department of Medicine, 200 Elizabeth Street, Suite RFE 3-805, Toronto, ON, M5G 2C4

\begin{abstract}
Two-thirds of out-of-hospital cardiac arrest patients, who survive to hospital admission, die in the hospital from neurological injuries related to cerebral hypoperfusion. Therefore, non-invasive real-time monitoring of the cerebral oxygen metabolism in cardiac arrest patients is extremely important. Hyperspectral near-infrared spectroscopy (hNIRS) is a non-invasive technique that measures concentrations of the key chromophores in the brain, such as oxygenated hemoglobin, deoxygenated hemoglobin, and cytochrome $\mathrm{C}$ oxidase (CCO), an intracellular marker of oxygen consumption. We tested hNIRS on 10 patients undergoing transcatheter aortic valve insertion, where rapid ventricular pacing (RVP) is required to temporarily induces sudden hypotension and hypoperfusion that mimics cardiac arrest. Using multidistance hNIRS, we found that significant CCO redox changes occurred only in patients' brains while tissue oxygen saturation changes in the brain were close to those in the extracerebral tissues during RVP. We have demonstrated that hNIRS is feasible and can detect sudden changes in cerebral oxygenation and metabolism in patients during profound hypotension.
\end{abstract}

Keywords: near infrared spectroscopy, cardiac arrest, cytochrome c oxidase, cerebral oxygen saturation, transcatheter aortic valve insertion.

*Thu Nga Nguyen, E-mail: nga.nguyen@ryerson.ca

\section{Introduction}

Out-of-hospital cardiac arrest occurs in over 350,000 people in North America and has a low survival rate of $<10 \%[65,66]$. Approximately two-thirds of out-of-hospital cardiac arrest patients who are resuscitated subsequently die in hospital due to neurological injuries, which result from prolonged hypoperfusion and ischemia during cardiac arrest and resuscitation [67,68]. Ischemic brain injury is a major cause of neurological injury where no flow or low flow states impair the transport of oxygen to the brain $[67,68]$. There is a need to monitor cerebral perfusion and 
oxygenation. The American Heart Association has acknowledged that brain injury after cardiac arrest should be a critical focus in clinical research [23]. Cerebral monitoring is essential to provide optimal care to decrease ischemic brain injury after cardiac arrest.

There is currently no standard in measuring cerebral perfusion and oxygenation during cardiac arrest resuscitation. Near infrared spectroscopy (NIRS) recently has been increasingly evaluated as a tool to measure cerebral oxygenation during and after cardiac arrest resuscitation [24]. NIRS is based on the measurement of the intensity of near-infrared light in the range 700-1100 nm that passes through the skin, skull, scalp and the brain, which depends on the absorption and scattering coefficients of the tissue. The major chromophores absorbing near-infrared light are oxygenated hemoglobin $\left(\mathrm{HbO}_{2}\right)$, deoxygenated hemoglobin $(\mathrm{Hb})$, fat, water, and cytochrome $\mathrm{C}$ oxidase $(\mathrm{CCO})$ $[69,70]$. The measurement of hemoglobin in NIRS has been widely used to determine the tissue oxygen saturation in the brain $\left(\mathrm{tSO}_{2}\right)$; however, $\mathrm{CCO}$ has been challenging to detect previously [70]. CCO plays the key role in the mitochondrial oxygen metabolism resulting in the synthesis of adenosine triphosphate (ATP). While the total tissue concentration of CCO does not rapidly change over time, the changes in the difference between the concentrations of the oxidized and reduced forms of $\mathrm{CCO}$ (redox) can be measured based on the property that oxidized and reduced forms of $\mathrm{CCO}$ have different shapes of their absorption spectra [70]. The main challenge in measuring cerebral $\mathrm{CCO}$ redox changes $(\Delta[\mathrm{CCO}])$ in adult humans is due the significantly lower average tissue concentration of CCO compared to hemoglobin [71]. Commercially-available NIRS devices employ a few isolated wavelengths of near-infrared light (multispectral NIRS or mNIRS) instead of the full light spectrum, which may not be quantitatively accurate to measuring hemoglobin and CCO changes compared to using the whole broadband spectrum (hyperspectral NIRS or hNIRS) [60-62,71]. 
Previous animal and human studies have shown that changes in $\Delta[\mathrm{CCO}]$ occurred during functional activations and when oxygen delivery was compromised [19,21,62,65,66]. NIRS [10, $62,67,68]$ studies on humans have established compelling evidence that $\Delta[\mathrm{CCO}]$ has clinical relevance as a metabolic status of the brain and measure of changes in cerebral oxygen delivery. Using NIRS simultaneously with transcranial Doppler ultrasonography as a surrogate measure of the cerebral blood flow and oxygen delivery, Tisdall et al [67] found a linear relationship between $\Delta[\mathrm{CCO}]$ and cerebral oxygen delivery during hypoxemia in healthy adult humans. Furthermore, in another study [68] the microdialysate lactate/pyruvate ratio (LPR) was considered as a marker of anaerobic metabolism, which decreases showing a negative correlation with the increases in $\Delta[\mathrm{CCO}]$. Holper showed that the respiratory challenges in response to hypocapnia and hypercapnia comparable to the $\Delta[\mathrm{CCO}]$, a hemodynamic signal, in terms of reliability [84]. Nosrati et al [62] found that cardiopulmonary resuscitation resulted in much higher increase in $\Delta[\mathrm{CCO}]$ than in the tissue oxygen saturation in a porcine model of cardiac arrest. Furthermore, small observational studies have shown that patients who survived after cardiac arrest had significantly higher cerebral oxygen saturation compared to those who died [87-89].

The objective of this study was to examine the feasibility of using hNIRS to detect sudden changes in cerebral oxygenation and metabolism in patients undergoing transcatheter aortic valve insertion (TAVI) [90]. During a TAVI procedure, rapid ventricular pacing (RVP) is used to decrease stroke volume during balloon valvuloplasty and valve implantation. RVP can be used to model cardiac arrest as it produces transient periods of low flow or no flow perfusion states. hNIRS measurements could help to develop and validate a novel technology to measure cerebral perfusion and metabolism in the cardiac arrest patients and to determine the real time cerebral response to cardiopulmonary resuscitation (CPR). 


\section{Materials and methods}

\subsection{TAVI patients and procedure}

The study was approved by the St. Michael's Research Ethics Board (REB \#14-397) and by the Ryerson University Research Ethics Board (REB \#2016-177).

Ten patients consented to NIRS monitoring prior to their TAVI procedures. NIRS monitoring was blinded to the treating physicians and did not alter treatment in any way. The TAVI procedure was performed by trained cardiothoracic surgeons. Patients were placed under general anesthesia and clinical management was at the discretion of the anesthesiologist and cardiac surgery team. Blood pressure and blood flow were significantly decreased during transient RVP via a temporary pacing wire to minimize left ventricular ejection and cardiac motion. This stabilized the valvuloplasty balloon during inflation and was momentarily induced in the patients for approximately 10-30 seconds.

\subsection{NIRS setup}

hNIRS cutSOm sensors (non-invasive stick pads) and mNIRS (Equanox 7600, Nonin, MI, USA) were placed on each patient's forehead for the entire TAVI procedure. The hNIRS sensor was placed over the left forehead and the mNIRS sensor was applied onto the right forehead. A band was applied across the forehead to ensure contact of both sensors. The Equanox 7600 utilizes four wavelengths $(730,760,810$ and $880 \mathrm{~nm})$ with four-channels and uses calculations based on the Beer-Lambert law to determine regional oxygen saturation (rSO2) [46]. The hNIRS experimental setup of the TAVI procedure is shown in Fig. 1. The spectra were collected at the sampling rate of $2 \mathrm{~Hz}$ by two fiber optic spectrometers: QE 65000 and USB 4000 (Ocean Optics, Dunedin, FL, USA) at $3 \mathrm{~cm}$ and $1 \mathrm{~cm}$, respectively to separate the extra-cerebral and cerebral measurements. 
Both of these spectrometers had their range from 650 to $1100 \mathrm{~nm}$. QE65000 had a high signal to noise ratio (1000:1 single acquisition) sufficient to measure light at $3 \mathrm{~cm}$ distance from the source. Two custom-made 2-meter long optical fiber bundles (each made of seven $0.5 \mathrm{NA}, 400 \mu \mathrm{m}$ core $\varnothing$ multimode polymer-clad fibers with broad UV/VIS/NIR spectral range of $400 \mathrm{~nm}$ to $2200 \mathrm{~nm}$ Thorlabs, NJ) connected spectrometers with the patient's head. The two other optical fiber bundles were used to connect the probe with a halogen lamp light source (Fiber-Lite Dc 950H Fiber Optic Illuminator, Dolan-Jenner, MA). The Spectra Suite (Ocean Optics, FL) software was used to collect the broadband continuous-wave hNIRS data from both spectrometers with dark-signal correction.

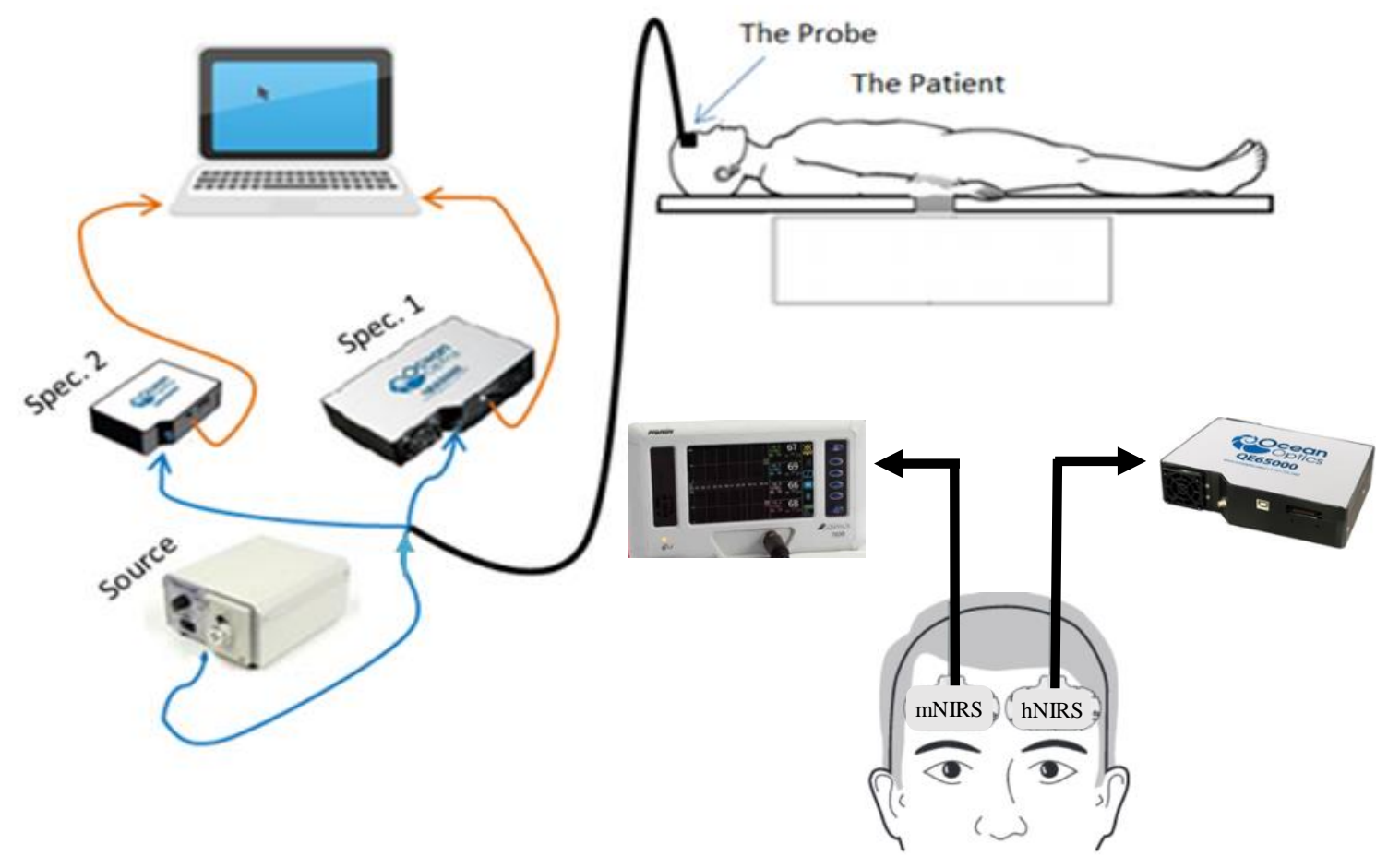

Fig. 1. TAVI experimental setup. 


\section{3 hNIRS data processing and analysis}

hNIRS data acquired at $1 \mathrm{~cm}$ and $3 \mathrm{~cm}$ channels represented the extra-cerebral layer (scalp + skull) and a combined extracerebral + cerebral tissue volume, respectively. The data processing algorithm of hyperspectral NIRS in our study was based on the analytical solution to the diffusion equation and implemented in MATLAB (Mathworks, MA, Version R2016b) [62]. The concentrations of chromophores were calculated by performing a non-linear square fitting the optical absorbance modeled as a function of the optical absorption coefficient $\mu_{a}(\lambda)$ and reduced scattering coefficient $\mu_{s}{ }^{\prime}(\lambda)$ in the spectral band of 650 to $1000 \mathrm{~nm}$. Changes in the hemoglobin concentrations [HbO2], [Hb] and $\mathrm{COO}$ redox were related to the changes in the optical absorbance as

$$
\Delta \mu_{a}(\lambda)=\Delta[H b] \varepsilon(\lambda)_{H b}+\Delta[H b O 2] \varepsilon(\lambda)_{H b O 2}+\Delta[C C O] \varepsilon(\lambda)_{c c o},
$$

where $\varepsilon(\lambda)_{x}$ were the spectra of the extinction coefficients of $\mathrm{HbO} 2, \mathrm{Hb}$, and $\mathrm{COO}$ redox [63].

Further details on recovering the absolute values and changes of chromophore concentrations from the hNIRS data can be found in [60].

As the fraction of oxygenated hemoglobin relative to the total hemoglobin in the blood, the cerebral tissue saturation of oxygen was calculated as:

$$
t \mathrm{SO}_{2}=\frac{\left[\mathrm{HbO}_{2}\right]}{\left[\mathrm{HbO}_{2}\right]+[\mathrm{Hb}]}(\%) .
$$

The analysis of TAVI data based on the hNIRS algorithm using homogenous model fit for the source-detector distances at $1 \mathrm{~cm}$ and $3 \mathrm{~cm}$ respectively, which were applied using Matlab (Mathworks, MA, Version R2016b).

\section{Results}


The results of the measurements are found in Fig. 2 and summarized in Table 1. Fig.2 showed changes in $[\mathrm{Hb}],\left[\mathrm{HbO}_{2}\right],\left[\mathrm{tSO}_{2}\right]$ and $\Delta[\mathrm{CCO}]$ measured and $1 \mathrm{~cm}$ and $3 \mathrm{~cm}$ by hNIRS in one TAVI patient during a time period including three RVP episodes indicated by the dashed lines. [ $\left.\mathrm{HbO}_{2}\right]$, $\left[\mathrm{tSO} \mathrm{S}_{2}\right]$, and $\Delta[\mathrm{CCO}]$ exhibited rapid drops while $[\mathrm{Hb}]$ showed increases during three RVP episodes lasted $20 \mathrm{~s}, 28 \mathrm{~s}$, and $24 \mathrm{~s}$, respectively; then all parameters changed towards their levels before RVP during approximately $90 \mathrm{~s}$. The longest (second) RVP caused significantly larger responses in all parameters.

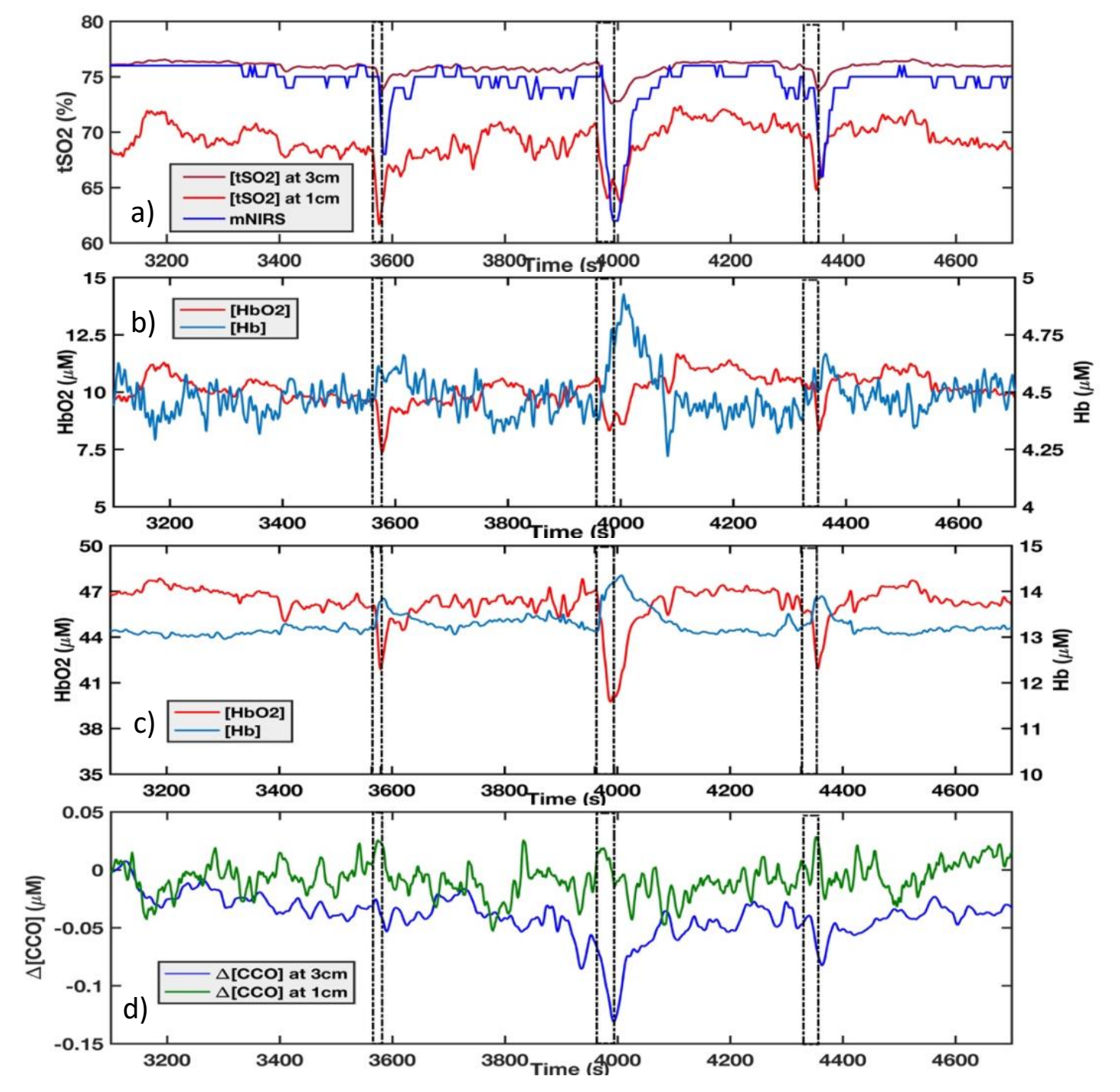


Fig. 2. Example of the time-course of the changes during a TAVI procedure (patient 10) for: a) [tSO ${ }_{2}$ ] for hNIRS at the $1 \mathrm{~cm}$, and $3 \mathrm{~cm}$ channels and mNIRS device, b) [Hb] and [HBO $\left.\mathrm{H}_{2}\right]$ changes at the $1 \mathrm{~cm}$ channel, $c$ ) at $3 \mathrm{~cm}$ channel, and d) $\triangle[C C O]$ at the $1 \mathrm{~cm}$ and $3 \mathrm{~cm}$ channels. Dashed lines indicate pacing episodes.

Fig. 2(a) shows $\mathrm{tSO}_{2}$ measured on the forehead by hNIRS at 1 and $3 \mathrm{~cm}$ channels and by mIRS. The time courses of all three $\left[\mathrm{tSO}_{2}\right]$ measurements are well consistent and showing similar trends during and after RVPs.

The baseline values in all mNIRS and hNIRS channels were close and normal (between $70 \%$ and $76 \%$ ). The magnitudes of changes in [ $\left.\mathrm{tSO}_{2}\right]$ during RVPs measured by the mNIRS and 1-cm hNIRS channels were comparatively close but both of them were significantly greater than the magnitudes of [ $\mathrm{tSO}_{2}$ ] changes measured at the $3 \mathrm{~cm}$ hNIRS channel.

Both $[\mathrm{Hb}]$ and $\left[\mathrm{HbO}_{2}\right]$ concentrations were lower at the $1 \mathrm{~cm}$ channel compared to those at the $3 \mathrm{~cm}$ channel as shown in Fig. 2 (b) and (c). The average changes during RVP for the TAVI patient 10 in $\left[\mathrm{HbO}_{2}\right]$ and $[\mathrm{Hb}]$ were approximately $5.2 \pm 1.97 \mu \mathrm{M}$ and $0.92 \pm 0.26 \mu \mathrm{M}$ at the $3 \mathrm{~cm}$ channel while at the $1 \mathrm{~cm}$ channel they were $2.3 \pm 2.3 \mu \mathrm{M}$ and $0.28 \pm 0.33 \mu \mathrm{M}$, respectively.

$\Delta[\mathrm{CCO}]$ (Fig. 2 (d)) decreased significantly at the $3 \mathrm{~cm}$ channel during the second and third RVPs, while $\Delta[\mathrm{CCO}]$ at the $1 \mathrm{~cm}$ channel did not show decreases during RVP, but showed random changes close to baseline (zero) level during the entire observation period.

The measurements of $\left[\mathrm{tSO}_{2}\right]$ and $\Delta[\mathrm{CCO}]$ for all 10 TAVI patients are summarized in Table 1. The table includes changes in [ $\mathrm{tSO}_{2}$ ] during RVPs at the $1 \mathrm{~cm}$ and $3 \mathrm{~cm}$ hNIRS channels and the mNIRS channel, as well as the $\Delta[\mathrm{CCO}]$ responses during RVPs. Significant $\Delta[\mathrm{CCO}]$ responses were measured during $13 \mathrm{RVP}$ episodes in eight patients. No significant $\Delta[\mathrm{CCO}]$ changes were detected in the $1 \mathrm{~cm}$ channel in any of the patients. Alternatively, the average $\Delta[\mathrm{CCO}]$ drop was $0.11 \pm 0.12 \mu \mathrm{M}$. The correlation between $\left[\mathrm{tSO}_{2}\right]$ and $\Delta[\mathrm{CCO}]$ in the $3 \mathrm{~cm}$ channel was $0.708 \pm$ 0.010. In all patients $\left[\mathrm{tSO}_{2}\right]$ responses to RVPs were similar to those shown in Fig. 2. [tSO $\mathrm{tS}_{2}$ at the 
$1 \mathrm{~cm}$ and mNIRS channels were significantly greater compared to the changes at the $3 \mathrm{~cm}$ channel.

On average, changes in $\left[\mathrm{tSO}_{2}\right]$ at $1 \mathrm{~cm}$ channel dropped by $6.95 \pm 0.79 \%$, while the mean drop in $\left[\mathrm{tSO}_{2}\right]$ at $3 \mathrm{~cm}$ channel was $2.96 \pm 0.30 \%$.

Table 1. The hNIRS-measured cerebral hemodynamics and metabolism for the $1 \mathrm{~cm}$ and $3 \mathrm{~cm}$ channels during the ventricular pacing for TAVI patients. The number of RVP episodes for each patient corresponds to the number of positive or negative response detections indicated as ' $\mathrm{Y}$ ' and ' $\mathrm{N}$ ', respectively.

\begin{tabular}{|c|c|c|c|c|c|c|}
\hline \multirow{2}{*}{$\begin{array}{c}\text { Patient \# } \\
\text { Patient } \\
\text { ID }\end{array}$} & \multicolumn{4}{|c|}{$3 \mathrm{~cm}$ channel } & \multicolumn{2}{|c|}{$1 \mathrm{~cm}$ channel } \\
\hline & $\begin{array}{c}\text { CCO } \\
\text { response } \\
\text { detected }\end{array}$ & $\Delta\left[\mathbf{t S O}_{2}\right] \%$ & $\Delta[\mathrm{CCO}] \boldsymbol{\mu M}$ & $\begin{array}{c}\text { STD } \\
\mu \mathrm{M}\end{array}$ & $\begin{array}{c}\text { CCO } \\
\text { response } \\
\text { detected }\end{array}$ & $\Delta\left[\mathrm{tSO}_{2}\right] \%$ \\
\hline 1 & $\mathrm{Y}$ & -1.40 & -0.070 & 0.0142 & $\mathrm{~N}$ & -4.30 \\
\hline 2 & $\mathrm{Y}$ & -2.10 & -0.050 & 0.0185 & $\mathrm{~N}$ & -10.0 \\
\hline 3 & $\begin{array}{l}\mathrm{Y} \\
\mathrm{Y}\end{array}$ & $\begin{array}{l}-3.40 \\
-1.30\end{array}$ & $\begin{array}{l}-0.200 \\
-0.090\end{array}$ & 0.0529 & $\begin{array}{l}\mathrm{N} \\
\mathrm{N}\end{array}$ & $\begin{array}{l}-16.0 \\
-5.00\end{array}$ \\
\hline 4 & $\mathrm{~N}$ & -2.40 & 0 & 0.0230 & $\mathrm{~N}$ & -10.0 \\
\hline 5 & $\mathrm{~N}$ & -2.80 & 0 & 0.0353 & $\mathrm{~N}$ & -5.20 \\
\hline 6 & $\begin{array}{l}\mathrm{Y} \\
\mathrm{Y}\end{array}$ & $\begin{array}{l}-5.20 \\
-6.00\end{array}$ & $\begin{array}{l}-0.150 \\
-0.500\end{array}$ & 0.0668 & $\begin{array}{l}\mathrm{N} \\
\mathrm{N}\end{array}$ & $\begin{array}{l}-9.70 \\
-12.0\end{array}$ \\
\hline 7 & $\begin{array}{l}\mathrm{Y} \\
\mathrm{Y}\end{array}$ & $\begin{array}{l}-4.00 \\
-2.70\end{array}$ & $\begin{array}{l}-0.080 \\
-0.170\end{array}$ & 0.0625 & $\begin{array}{l}\mathrm{N} \\
\mathrm{N}\end{array}$ & $\begin{array}{l}-4.60 \\
-4.10\end{array}$ \\
\hline 8 & $\begin{array}{l}\mathrm{Y} \\
\mathrm{Y}\end{array}$ & $\begin{array}{l}-1.90 \\
-3.70\end{array}$ & $\begin{array}{l}-0.060 \\
-0.120\end{array}$ & 0.0391 & $\begin{array}{l}\mathrm{N} \\
\mathrm{N}\end{array}$ & $\begin{array}{l}-3.10 \\
-4.50\end{array}$ \\
\hline 9 & $\begin{array}{l}\mathrm{N} \\
\mathrm{Y}\end{array}$ & $\begin{array}{l}-2.50 \\
-2.60\end{array}$ & $\begin{array}{c}0 \\
-0.110\end{array}$ & 0.0267 & $\begin{array}{l}\mathrm{N} \\
\mathrm{N}\end{array}$ & $\begin{array}{l}-5.70 \\
-6.00\end{array}$ \\
\hline 10 & $\begin{array}{l}\mathrm{N} \\
\mathrm{Y} \\
\mathrm{Y}\end{array}$ & $\begin{array}{l}-2.10 \\
-3.60 \\
-2.70\end{array}$ & $\begin{array}{c}0 \\
-0.070 \\
-0.040\end{array}$ & 0.0165 & $\begin{array}{l}\mathrm{N} \\
\mathrm{N} \\
\mathrm{N}\end{array}$ & $\begin{array}{l}-6.50 \\
-6.00 \\
-5.50\end{array}$ \\
\hline $\begin{array}{c}\text { Average } \\
\text { data }\end{array}$ & NA & $-2.96 \pm 0.30$ & -0.101 & 0.0350 & NA & $-6.95 \pm 0.79$ \\
\hline
\end{tabular}

*STD: standard deviation of [CCO].

*Y: Yes, a significant drop in [CCO] was detected.

*N: No significant drop in $[\mathrm{CCO}]$ was detected.

\section{Discussion}


Using hNIRS, we measured cerebral oxygen saturation and metabolism in TAVI patients during the transient RVP, which is our patient model of "controlled cardiac arrest". Overall, we observed a significant impact of RVPs on the various changes occurred in cerebral concentrations of $[\mathrm{Hb}]$, $\left[\mathrm{HbO}_{2}\right],\left[\mathrm{tSO}_{2}\right]$ and $\mathrm{CCO}$ redox. In particular, due to profound hypoperfusion during RVPs, the concentration of $[\mathrm{Hb}]$ increased while $\left[\mathrm{HbO}_{2}\right]$ and $\left[\mathrm{tSO}_{2}\right]$ decreased in the brain is consistent with previously published studies $[1,34,73,90]$. The information from the hemoglobin signals provided the intravascular oxygen status, while $\Delta[\mathrm{CCO}]$ reflected changes in the intracellular oxygen consumption by cerebral neurons, which has been extensively investigated as a non-invasive marker of cellular oxygen metabolism $[1,19,66,71]$.

The $1 \mathrm{~cm}$ channel reflected the status of the non-cerebral tissue such as scalp and skull, while the $3 \mathrm{~cm}$ channel interrogated both the non-cerebral tissue and the cerebral cortex. In the brain, the concentration of CCO was higher than in the scalp and skull $[67,73,75,76]$. The presence of $\Delta[\mathrm{CCO}]$ responses to RVPs at the large source-detector distance $(3 \mathrm{~cm})$ and the absence of them at the small distance $(1 \mathrm{~cm})$ confirmed the brain-specificity of $\Delta[\mathrm{CCO}] . \Delta\left[\mathrm{tSO}_{2}\right]$ did not show the same consistent behavior.

In two patients $\Delta[\mathrm{CCO}]$ were not detected in both short and long-distance hNIRS channels. A possible explanation could be due to the shrinking of the brain in elderly humans [77], leading to increased distance from the brain parenchyma and skull that is separated by cerebrospinal fluid (CSF) resulting in a low reflection of light from the brain. According to Okada et al [29], the presence of the cerebrospinal fluid has a substantial effect on the penetration of the near-infrared light into the brain. Numerous studies have reported that aging commonly causes atrophy of the brain, as significant parenchyma loss of both gray and white matter could be seen while cerebrospinal fluid (CSF) increases in volume [77-78]. Furthermore, it is known that on average 
the differential path-length factor increases with the human age [2] due to the higher scattering in the extra-cerebral tissue, thus overall penetration into the brain decreases with the age. However, age-related changes can be inconsistent between patients.

We found that the changes in CCO responses to the transient RVPs during the TAVI depended on the source-detector separation, which reflected the difference of CCO concentration in the extracerebral and cerebral tissues. Furthermore, the values of $\Delta\left[\mathrm{tSO}_{2}\right]$ measured by hNIRS at the longer source-detector spacing $(\sim 2.96 \%)$ were lower compared to those at the shorter sourcedetector distance $(\sim 6.95 \%)$. These differences as well as the differences in the absolute values of hemoglobin concentrations could be due to the fact that a significant portion of the volume interrogated by the $3 \mathrm{~cm}$ channel was within the brain, which had significantly different optical properties compared to the scalp. Such a strong heterogeneity could strongly bias the quantitative accuracy of the determination of cerebral tSO2 changes by hNIRS. The measurements of cerebral tSO2 by the mNIRS monitor could be more accurate due to the calibration of Equanox 7600 for the influence of the skull [46]. On the other hand, the ranges of $\Delta[\mathrm{tSO} 2]$ measured by mNIRS and by hNIRS at $1 \mathrm{~cm}$ were close. This showed that unlike $\Delta[\mathrm{CCO}]$, changes in tSO2 during RVPs could be non-specific for the brain.

Our results agreed with prior reports of near-infrared signals in each layer of the head tissue by using magnetic resonance imaging [79], time-resolved spectroscopy measurement [80], and Monte Carlo simulations [65]. These studies found a high correlation between hemodynamic changes with cerebral hemoglobin signal in the extracerebral tissues. The Monte Carlo simulations [65] results revealed that the largest amount of detected photons propagating into the brain arises from the brain cortex at a $30 \mathrm{~mm}$ source-detector separation. However, changes in $\Delta[\mathrm{CCO}]$ are less prone to the extracerebral contamination and is more specific to the mitochondrial oxygen 
concentration [74]. Our results agree with Kolyva et al [19], who found that the $\Delta[\mathrm{CCO}$ ] response was distinctly different from hemodynamic signal, such that there is a presence of a gradually increasing of its response with increasing source- detector distance in four different experimental prototypes: hypoxia, hyperoxia, hypocapnia and hypercapnia.

Even though the study has shown encouraging results on measuring $\Delta[\mathrm{CCO}]$ during TAVI using hNIRS, the study had several limitations. The biological and optical properties and the thickness of the non-cerebral tissue vary from patient to patient. The thickness of the non-cerebral tissue could not be determined noninvasively in this specific clinical setting. Due to the extracranial contamination, changes in $\left[\mathrm{tSO}_{2}\right]$ could be lower at $3 \mathrm{~cm}$ than at $1 \mathrm{~cm}$. As the general thickness of the non-cerebral layer of human head could range between 10 and $15 \mathrm{~mm}$, the biological and optical properties of the extracerebral tissue in TAVI patient varied due to different factors such as age, gender, etc. Hence, the characteristics of the extracerebral tissue need to be determined to estimate the cerebral changes with better quantitative accuracy. Future studies are needed to determine the optimal distance between optodes to accurately measure $\Delta[\mathrm{CCO}]$.

Further research is required to evaluate the use of hNIRS in prolonged hypoperfusion such as cardiac arrest patients. Nevertheless, the objective of this study has shown the feasibility of hNIRS to measure cerebral oxygen saturation and $\Delta[\mathrm{CCO}]$ in controlled model of the cardiac arrest.

\section{Conclusion}

We have shown that hNIRS is sensitive to sudden changes in $\left[\mathrm{tSO}_{2}\right],[\mathrm{Hb}],\left[\mathrm{HbO}_{2}\right], \mathrm{tHb}$ and $\Delta[\mathrm{CCO}]$ during profound hypoperfusion in TAVI patients. Changes in $\mathrm{CCO}$ redox measured by hNIRS was a more specific and reliable marker of cerebral status than $\mathrm{tSO} 2$. 


\section{Disclosures}

The authors have no relevant financial interests in this article and no potential conflicts of interest to disclose.

\section{Acknowledgments}

We gratefully acknowledge the physicians and patients from St. Michael's Hospital for their support in this study ${ }^{41}$. This study was supported by the Heart and Stroke Foundation of Canada Emerging Research Leaders Initiative grant and the St. Michael's Foundation Translational Innovation Fund. S.L. is Network Investigator of the Cardiac Arrhythmia Network of Canada (CANet) and is supported by a Heart and Stroke Foundation of Canada Ontario Clinician-Scientist award. 


\section{Chapter 3}

\section{Additional Methods, Results and Discussion}

\section{1 - Breath holding measurement}

\subsection{1 - System overview and respiratory challenges}

Breath holding method or breath holding index (BHI) is known as the risk marker for stroke in asymptomatic carotid stenosis which defined with transcranial Doppler (TCD) $[81,82]$. Given that TAVI is the treatment of severe aortic stenosis without having a standard open heart surgery, this non-aggressive, real-time BHI method can also be used to study the cerebral hemodynamics and cellular metabolism of the brain without any required data from the patients from the hospital settings [83]. Having a stable concentration and a higher brain- specificity compared to hemoglobin, the redox state CCO can be tested in a healthy population to estimate its reliability. With this breath holding method, two abnormal respiratory conditions can be achieved to systemically manipulate cerebral oxygenation via hypo and hypercapnia $[84,85]$. Holper et al explained hypercapnia challenges as the reduced of carbon dioxide in the blood by inducing hyperventilation while hypercapnia increased carbon dioxide in the blood which was induced by breath holding of 20 seconds and normal breathing of 40 second [85]. Alternatively, the hNIRS algorithm could be used to achieve these two reparatory challenges to show the relative changes of hemoglobin and oxCCO. Three different spectrometers USB4000, QE65000 and AVANTES were used simultaneously at three different source-detector distances $10 \mathrm{~mm}, 30 \mathrm{~mm}$ and $40 \mathrm{~mm}$ utilizing the two- layer model approach at different optode separations. 


\subsection{2 - Experimental protocol and setup}

It was concentrated on assessing the dynamic changes in the chromophores in the brain to reflect the consumption and oxygen delivery in five healthy subjects ( 1 female and 3 males, ages of 19 to 55) underwent NIRS examination during breath-holding measurement. All participants without any previous training in the performance of breathing procedure were comfortably positioned on the chair with sudden command performed three - 30 seconds of breath holdings, along with 90 seconds rest interval in between, and 270 seconds of relaxing breathing time. Both Equanox 7600 NONIN and hNIRS sensors attached on the left and right side of the forehead, respectively with the black headband. Healthy subjects were instructed to remain stable as possible during the measurements to minimize the effect of motion artifacts on hNIRS data and prevented the signal detection of the surrounding light. The experiment protocol was within 10 minutes, which at 1, 3, and 5 minutes do the breath hold of 30 seconds with the alternate period of deep breathing in between while the last 4 min 30 seconds the subject performed normal, relaxed breathing as the resting- state period. Prior to each challenge, the participants were instructed to exhale as much as possible before the breath holding cycle started to avoid inhaling a larger volume of air than the volume of the normal breath cycle.

\subsection{3 - Data analysis}

All the data were performed using MATLAB (Mathworks, MA, Version R2016b). This code was based on the broadband NIRS range from 710nm to $950 \mathrm{~nm}$ and multiple wavelength combinations of our proposed algorithm using homogenous model fit. For the assessment of the combinations 
of wavelengths, the 3,4,5, and 8 - optimized wavelengths combination for NIRS in the brain region were given from the previous study by Arifler et al [20]. They were compared with the hNIRS measurements during the breath holding procedure. The three hypercapnia intervals were measured within 30 seconds of breath holding from the baseline.

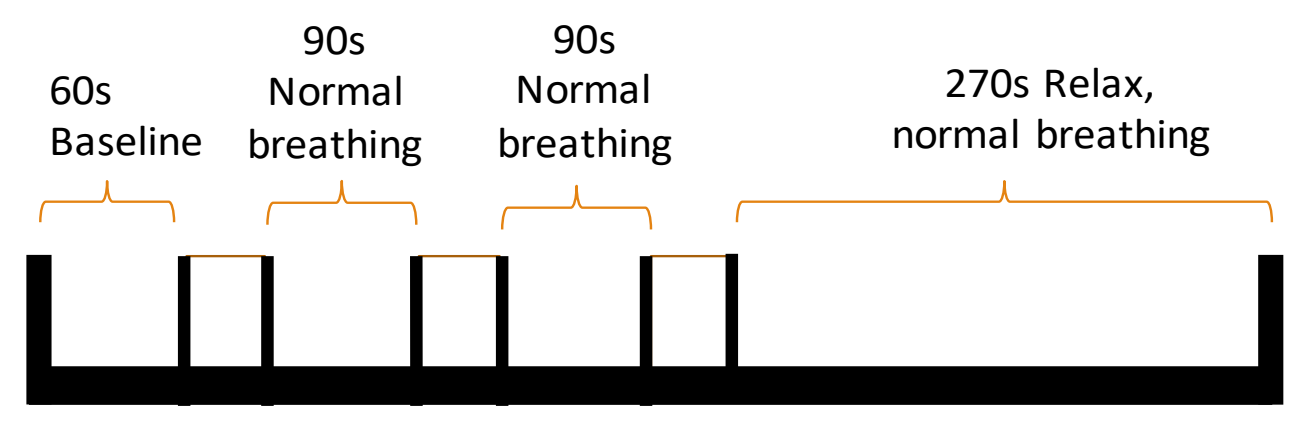

a)

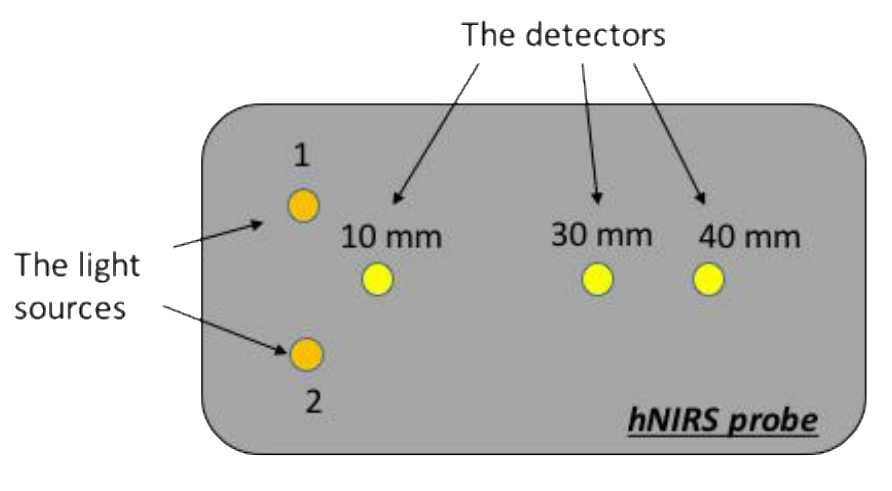

b)

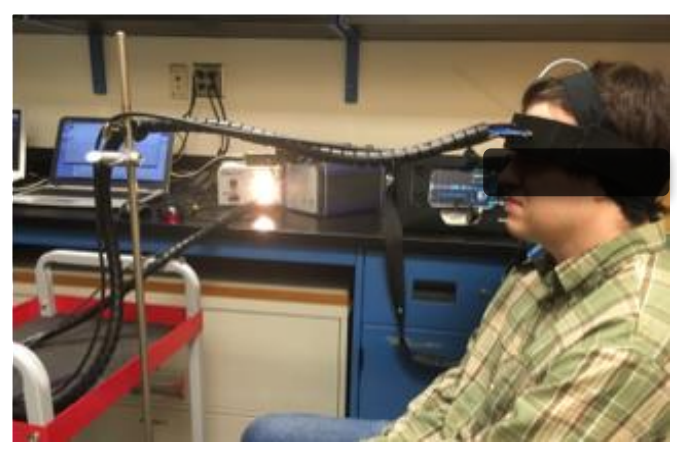

c)

Figure 11. a, The experimental model, b) The probe for the hNIRS algorithm and $c$, the experimental setup of hNIRS at 10mm, 30mm and 40mm for USB 4000, QE 65000, and Avantes.

\section{2 - Results and Discussion}

\subsection{1 - Results of 2 layer-model and breath holding measurements}

The results and discussion on examining the feasibility of using the hNIRS algorithm to detect changes in cerebral oxygenation and metabolism in patients undergoing TAVI have described in 
Chapter 2. This section covered the results of the 2-layer model approach of TAVI patients and breath holding measurements based on homogenous approach on healthy subjects.
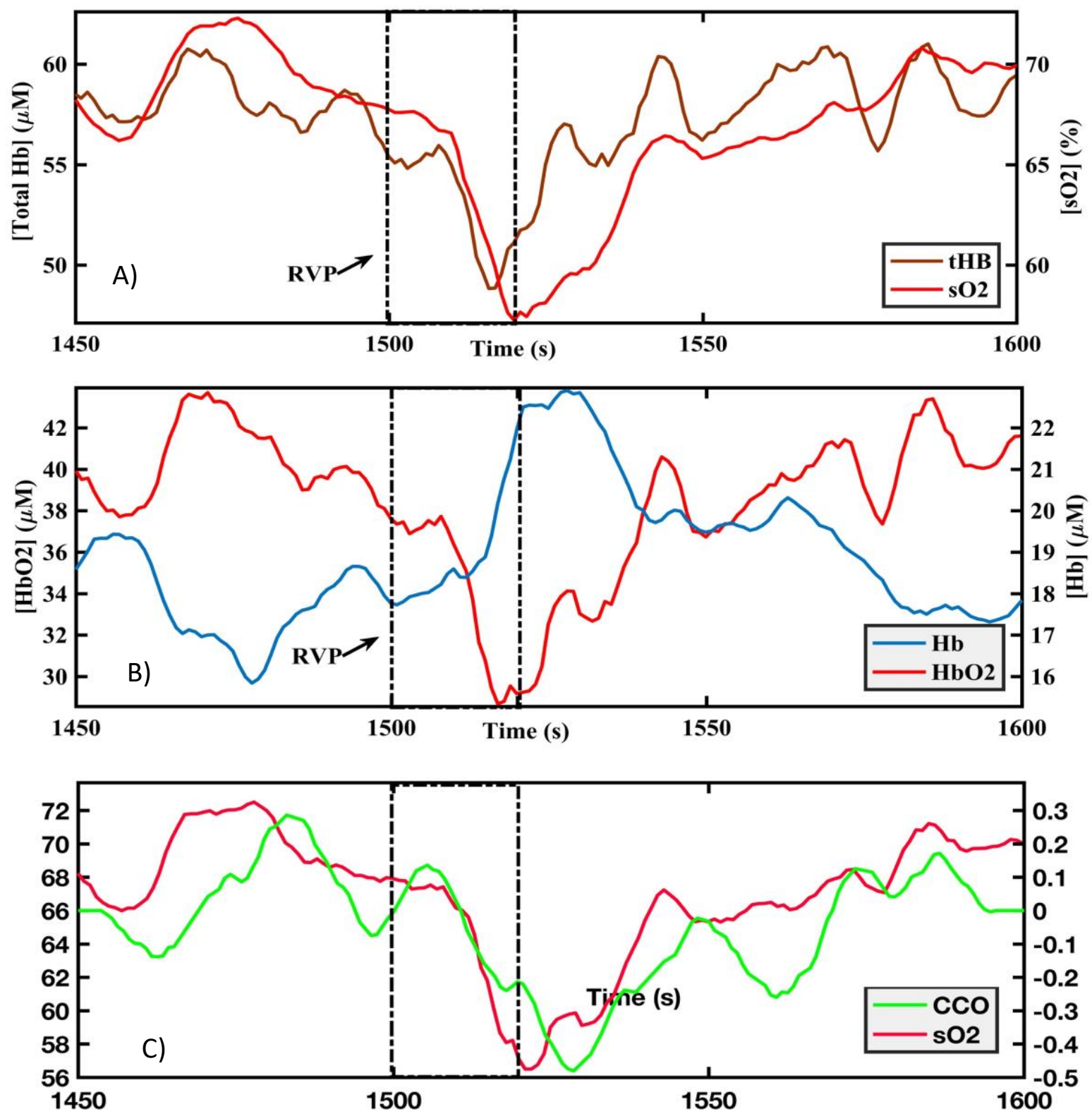

Figure 12. Time course series of hNIRS-measured changes of TAVI patient during RVP are shown in a) [Hb] and [HBO2], b) Total Hb and [StO2], c) $\triangle$ [CCO] and [StO2]. Ventricular pacing indicated in the dot-dashed boxes. The plot is shown for an example participant. 
From the simultaneous measurements on the extracranial and intracranial tissues, the changes of $[\mathrm{Hb}],\left[\mathrm{HbO}_{2}\right], \mathrm{tHb}$ and $\left[\mathrm{StO}_{2}\right]$ during ventricular pacing of one TAVI patient were presented in Figure 3.2 in 20 seconds of duration time. Their time course series of 1500 seconds shows the rapid significant changes were induced during RVP phase. While $\left[\mathrm{HbO}_{2}\right], \mathrm{tHb},[\mathrm{CCO}]$ and $\left[\mathrm{StO}_{2}\right]$ decreased sharply and consistency at the similar trends, $[\mathrm{Hb}]$ displayed in the opposite direction by showing a sharp peak to determine the significant increasing during RVP. Before the RVP time, $[\mathrm{HbO} 2], \mathrm{tHb},[\mathrm{CCO}]$ and $\left[\mathrm{StO}_{2}\right]$ gradually decreased with a few small peaks until there were drastic drops during RVP, then rapidly recovered back. Respectively, the changes in [ $\left.\mathrm{HbO}_{2}\right],[\mathrm{Hb}]$, [CCO] and [StO 2$]$ were approximately $8 \mu \mathrm{M}, 6.5 \mu \mathrm{M}, 0.55 \mu \mathrm{M}$ and $11 \mu \mathrm{M}$ during RVP.
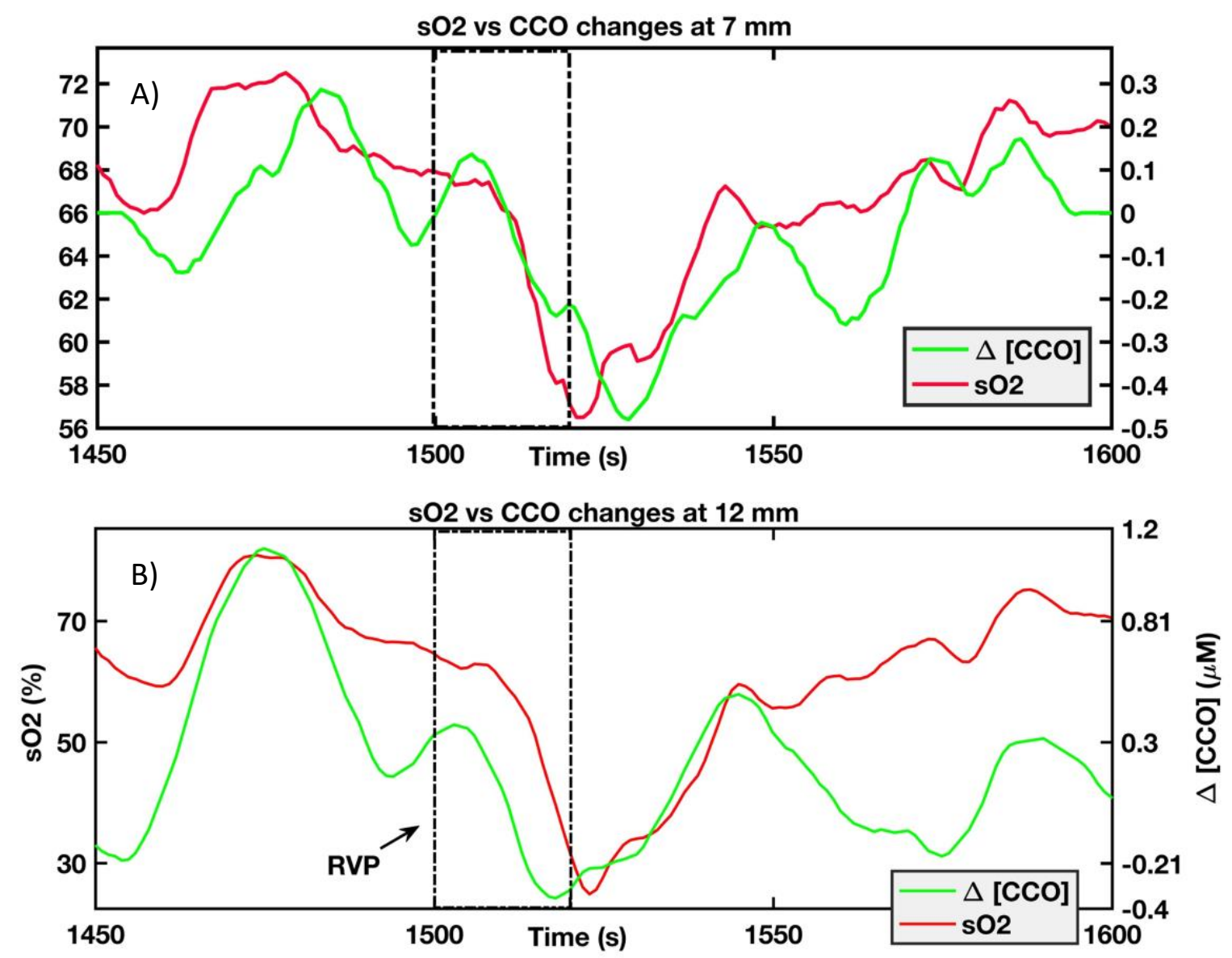

Figure 13. The top layer thickness measurements of hNIRS for TAVI patients. 
Figure 3.3 shows the time course series to investigate the thickness measurements of hNIRS for the extra cerebral tissue at $7 \mathrm{~mm}$ and $12 \mathrm{~mm}$ throughout the changes in oxygen saturation and [CCO]. There was a relative trend of decreasing from the baseline until the end of the RVP, then gradually increased back to the baseline values in both graphs. However, at $12 \mathrm{~mm}$ thickness the changes in both tSO2 and [CCO] have significant lower drops. There is also a time delay of $\Delta[\mathrm{CCO}]$ after the RVP episode at $7 \mathrm{~mm}$ thickness, but this did not show the same behavior at $12 \mathrm{~mm}$.

A)

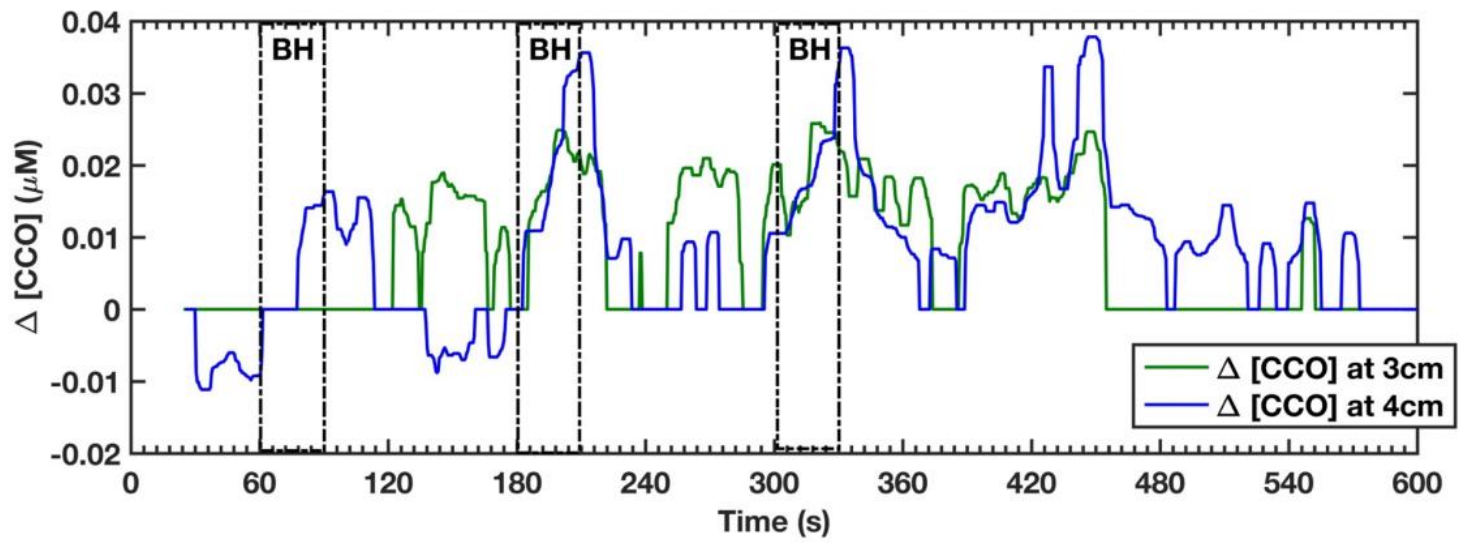

B)

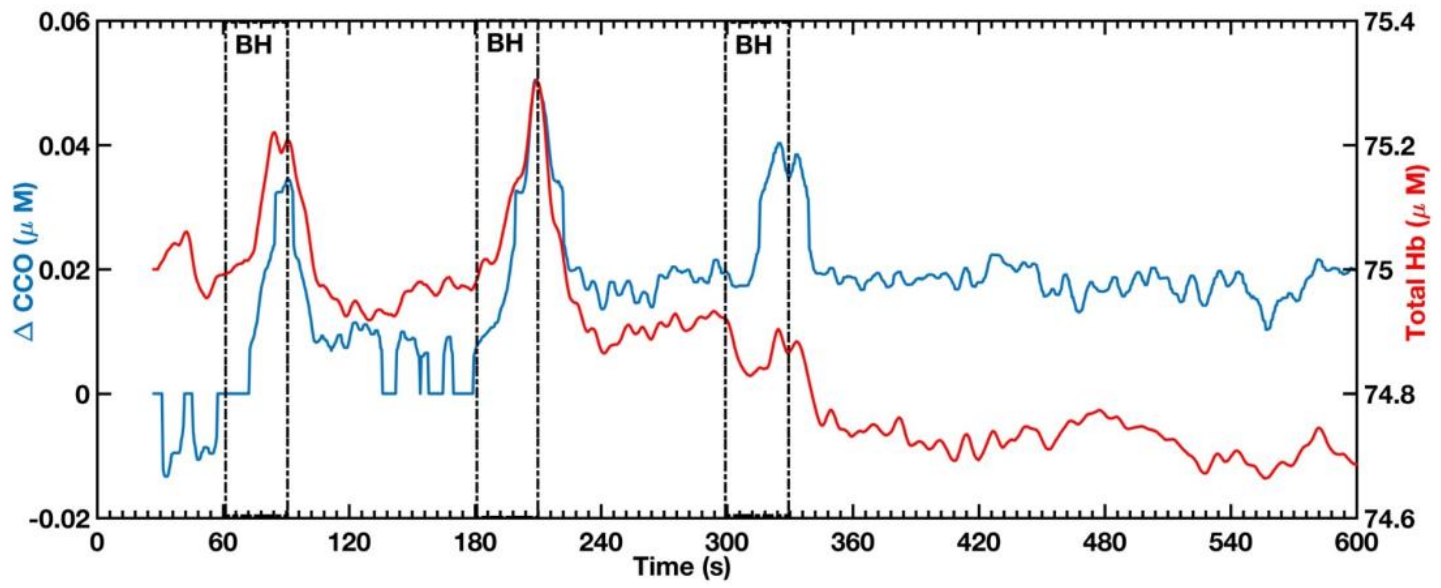

Figure 14. hNIRS measurements of a) $\triangle[C C O]$ at 3 , and $4 \mathrm{~cm}$, and b) $\triangle[C C O]$ and total hemoglobin during breath holding (BH) measured by hNIRS at the optode separation of $4 \mathrm{~cm}$. The plot is shown for an example participant. 
Figure 3.4 shows the changes in the cytochrome $C$ oxidase $(\Delta[\mathrm{CCO}])$ at $3 \mathrm{~cm}$ and $4 \mathrm{~cm}$ optode separations and $\Delta[\mathrm{CCO}]$ versus total hemoglobin concentrations (Total $\mathrm{Hb}$ ) during breath holding (BH) for a healthy subject measured by hNIRS. From Figure $3.4 \boldsymbol{a}$, the $\Delta[\mathrm{CCO}]$ at $1 \mathrm{~cm}$ channel was ignored since it did not show any chances and the values were completely at zero. It shows a great correlation between $3 \mathrm{~cm}$ and $4 \mathrm{~cm}$ channels during $\mathrm{BH}$. At $4 \mathrm{~cm}$ channel, the changes in $[\mathrm{CCO}]$ is more significant as the magnitude of the changes in $[\mathrm{CCO}]$ response were higher during $\mathrm{BH}$.

Similarly, the time course series of both are consistent during three $\mathrm{BH}$ intervals for $\Delta[\mathrm{CCO}]$ and Total $\mathrm{Hb}$ as shown in Figure 3.4 b. It shows relative changes in response to $\mathrm{BH}$ for all signals and the returns close to baseline values after each episode. From the baseline, Total $\mathrm{Hb}$ started to drastically increase at the beginning of the breath hold then quickly decreased down to the baseline as soon as the $\mathrm{BH}$ episode was done. Similarly, $\Delta[\mathrm{CCO}]$ had the consistent trend of increase during the HB and achieved a significant drop quickly after the 30 second of breath holding. Within the first and second of 90 seconds of resting time, both Total $\mathrm{Hb}$ and $\Delta[\mathrm{CCO}]$ went back to the baseline level. Within the last 270 second of regular breathing, the baseline of $\Delta[\mathrm{CCO}]$ gradually decreased from each baseline interval which leads to the changes in [CCO] did not reach to the baseline before the $\mathrm{BH}$ procedure. However, the baseline of Total $\mathrm{Hb}$ after the $\mathrm{BH}$ briefly higher compared to the baseline before the $\mathrm{BH}$ episodes. This created the three significant peaks in the plots in the graph of Total $\mathrm{Hb}$ and $\Delta[\mathrm{CCO}]$ indicating three intervals of the breath holding.

The comparison of the changes in the cytochrome $\mathrm{C}$ oxidase redox measured by our proposed mNIRS algorithm using 3, 4, 5 and 8 - optimized wavelengths combinations based on the previous study on the animal model and mNIRS -8 wavelength combinations versus hNIRS plots can be shown as in Figure $3.5 \boldsymbol{a}$ and $\boldsymbol{b}$. It shows the time course series of the breath holding of a healthy 
subject within 3 intervals. Alternatively, since 8-wavelength combination has the lowest percentage of error as $2 \%$, Figure 3.5 , displays that the response of $\Delta[\mathrm{CCO}]$ at 8 wavelength combination is the most significant compared to the 3,4 , and 5 wavelength combinations signals. In addition, there is a notable consistency in the quick peaks of $\Delta[\mathrm{CCO}]$ during the $\mathrm{BH}$ period in Figure 3.5 b. Although, hNIRS measurement shows a significant incline in changes of [CCO] than mNIRS measurement during the $\mathrm{BH}$ episodes. Consistently, the post baseline in both measurements of hNIRS and mNIRS are briefly higher compared to the baseline before the $\mathrm{BH}$ procedure.

A)

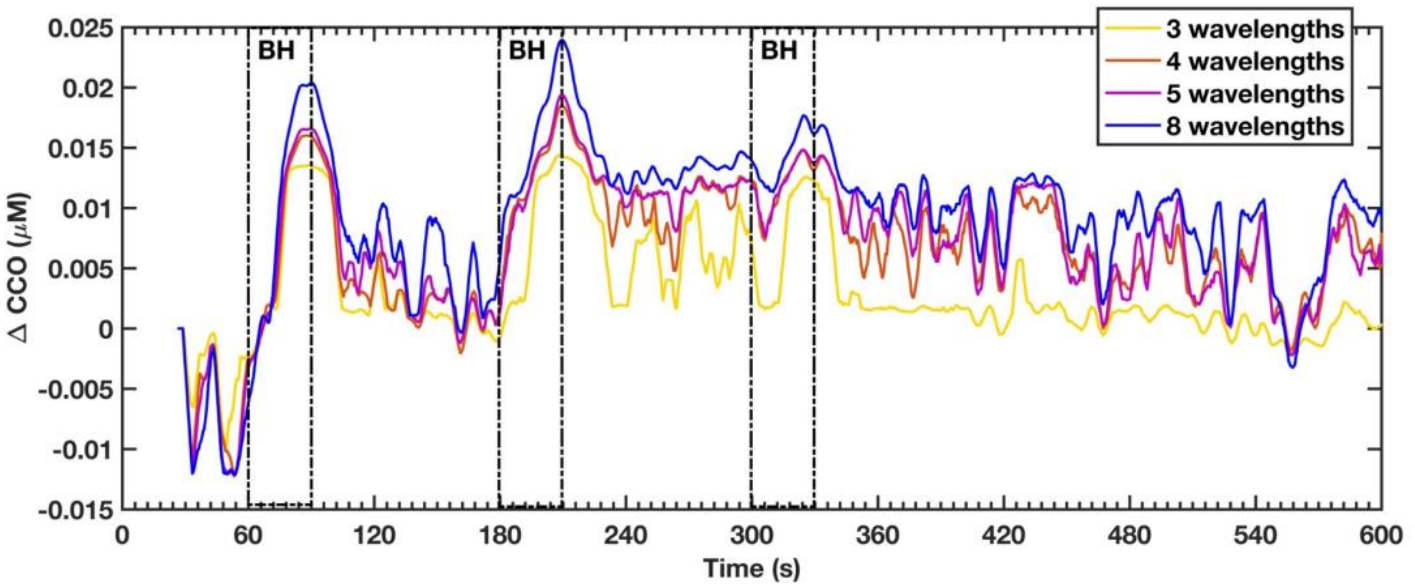

B)

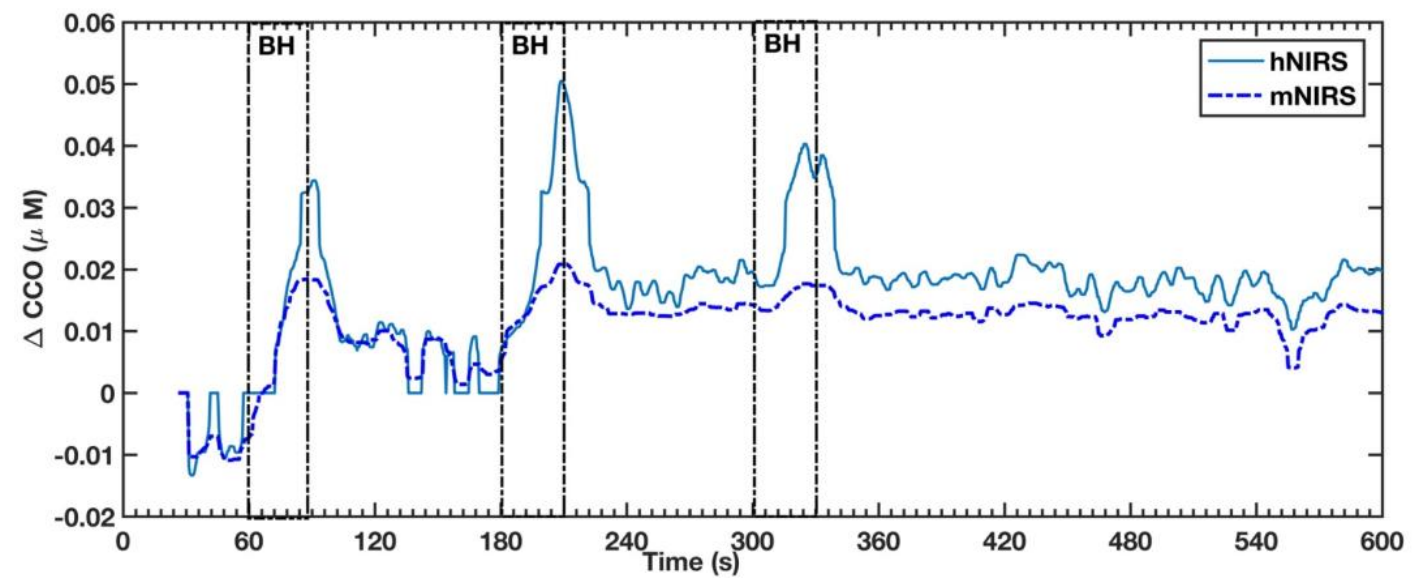

Figure 15. Changes in the cytochrome C oxidase redox state measured by mNIRS a) using 3,4,5 and 8-optimized wavelengths and b) mNIRS at 8-selected wavelength combination and hNIRS at the optode separation of $4 \mathrm{~cm}$. The plot is shown for an example participant. 


\subsubsection{Discussion of 2 layer-model and breath holding measurements}

This study helps to determine the feasibility of using hyperspectral near infrared spectroscopy in patients undergoing the TAVI procedure which mimics cardiac arrest in the prehospital setting. It can be found that there is a significant measurement of oxygen saturation during transient rapid ventricular pacing of TAVI treatment strategy was mainly from extra- cerebral tissues. Under the influence of the 2-layer model technique in the broadband spectroscopy hNIRS, the changes in oxygen saturation and $[\mathrm{CCO}]$ were successfully achieved. There was a significant impact of rapid ventricular pacing system on the various changes occurred in cerebral $[\mathrm{Hb}],\left[\mathrm{HbO}_{2}\right], \mathrm{tHb},\left[\mathrm{StO}_{2}\right]$ and [CCO], which give reasonable and consistent values with previous studies [63,70-71].

From a previous animal study where the authors also used hNIRS technique to measure $\left[\mathrm{StO}_{2}\right]$ invasively (dura) and semi-invasively (skull) during cardiac arrest, it showed that there was a temporal delay in $[\mathrm{CCO}]$ compared to the level of cerebral $\left[\mathrm{HBO}_{2}\right],[\mathrm{HB}]$, and $\left[\mathrm{StO}_{2}\right]$ during hypoxic states may due to the ability of neurons to extract oxygen $[62,74]$. This agrees to our hNIRS clinical setting where we also found that there was a delay in increasing of [CCO] within RVP. This indicates the significant correlation between cytochrome $\mathrm{c}$ oxidase with impaired mitochondrial respiration in cardiac arrest.

We found that in the homogeneous layer condition in the brain, the results of the changes in oxygen saturation at distance $1 \mathrm{~cm}$ (extra cerebral) using hNIRS was more consistent with mNIRS; however, using the 2-layer model, the response of the changes in [CCO] was predominantly at $3 \mathrm{~cm}$ of hNIRS data. Relatively, the longer optode decreases the transmission of the light through the tissue and causes a decline result. 
Furthermore, we also observed that the changes in oxygen saturation scale with the thickness of the top layer (extra cerebral tissue) in the 2-layer model hNIRS algorithm. With the thicker layer of extra cerebral tissue, the less near-infrared signal can be detected. Therefore, the drop of $\left[\mathrm{StO}_{2}\right]$ during RVP at a thicker extra cerebral tissue would have a lower value. Okada and Young reported that the sensitivity of cerebral NIRS signal was affected by the thickness of the skull layer [86]. The values of $[\mathrm{CCO}]$ is less prone to extracranial contamination than cerebral hemoglobin signal since the concentration of $[\mathrm{CCO}]$ is mainly present in cerebral tissue compared to cerebral component [19].

Both general anesthesia and sedation were used in TAVI treatment, however, it was verified that they did not have a statistically significant and clinical difference in term of cerebral desaturation, and only sedation was associated with respiratory events. In addition, the geometry of the measurement in terms of the source-detector distance could be one of the critical factors that influences the cerebral NIRS measurements.

The interpretation of hemodynamic changes shown in Figure 3.4 reflects the normal cerebrovascular reactivity to hypoxia which causes a significant prominent increase in the volume of the cerebral blood with the increase in mitochondrial cellular metabolism during the breath holding episodes. These results agree with the previous finding for healthy adult volunteers using NIRS measurements $[83,85]$.

As the marker of mitochondrial health, Cytochrome $\mathrm{C}$ oxidase measurement derived from our proposed hNIRS algorithm can yield crucial information about cerebral metabolism. Reliability of the cellular measurements of the metabolic redox state of cytochrome $\mathrm{C}$ oxidase has been previously determined to be excellent in healthy adults by the assessment of the Intraclass Correlation Coefficients (ICCs) with confidence intervals based on the significant level of alpha 
as $\mathrm{p}<0.05$ (ICC $=0.8727-0.913)$ [85]. Furthermore, with the excellent ICC value of the hemodynamic measurements $(\mathrm{ICC}=0.80-0.84)$, the previous study suggest that the redox state of cytochrome $\mathrm{C}$ oxidase signal can be a reliable chromophore to be implemented effortlessly in both research and clinical setting under respiratory challenges.

Regarding the method of NIRS measurements, the study utilized an Equanox 76000 NONIN equipment which only used four discrete wavelengths (730, 760, 810, and $880 \mathrm{~nm}$, respectively) at $4 \mathrm{~cm}$ channel is better than at $3 \mathrm{~cm}$ channel for $\mathrm{CCO}$ measurement. The same hNIRS data set for an example participant was processed using multispectral algorithm using 8 isolated wavelengths combination $(784,800,818,835,851,868,881$, and 894 , respectively) for the $4 \mathrm{~cm}$ channel as shown in Figure 3.5b. The signal using broadband spectroscopy (hyperspectral algorithm) from Figure $3.4 \boldsymbol{b}$ is consistent with the signal from the multispectral algorithm of 8 selected wavelengths, where they both show significant incline peaks during $\mathrm{BH}$ periods. However, the measurements of $\triangle \mathrm{CCO}$ by the mNIRS monitor is not as significant as the hyperspectral and multispectral algorithm measurements. Notably, the measurement of the cerebral oxygen saturation by mNIRS method could be more accurate due to the calibration of Equanox 76000 for the influence of the skull [46]; however, the results of $\Delta[\mathrm{CCO}]$ at $4 \mathrm{~cm}$ channel which is for the brain region, show that out proposed algorithm was more sensitive to changes in [CCO] during BH.

Nevertheless, this study has been investigated successfully on the feasibility of hNIRS measurement not only on cardiac arrest, but also on the breath holding procedure which has comparable results with previous studies $[19,85]$. 
Chapter 4

Conclusions and Future work 


\section{Conclusions}

In my study, hNIRS provided the utility for monitoring changes in $\mathrm{tSO}_{2}, \mathrm{Hb}, \mathrm{HbO}_{2}$, $\mathrm{tHb}$ and of cytochrome $\mathrm{C}$ oxidase redox occurring during sudden profound hypoperfusion episodes in TAVI patients which mimics cardiac arrest. In addition, the changes in tissue oxygenation saturation measured by mNIRS during cardiac arrest mainly reflected changes in the extra cerebral tissue while the changes in cytochrome $\mathrm{C}$ oxidase occurred mainly in the brain. It also revealed the use of hNIRS in a 2-layer model of the human head where changes in all parameter scaled with the thickness of the extra cerebral layer and high correlation between $\mathrm{CCO}$ and impaired mitochondrial respiration in cardiac arrest.

My results from the breath holding experiment suggest a higher sensitivity in hNIRS measurements on the redox of cytochrome $\mathrm{C}$ oxidase in the cerebral region. In regards to the selected wavelengths of the multispectral NIRS optimized for the neonatal head in the animal study [20], mNIRS also can measure changes in CCO in adults, however the wavelength may need to be further optimized for the adult patient. Overall, my results contribute to the development of a new NIRS technology suitable for the emergency cardiac arrest treatment.

\section{Future work}

In the future, this study paves the way to new investigations and fills the knowledge gaps in the further research and clinical studies such as

- Further validation of the redox state of Cytochrome $\mathrm{C}$ oxidase study by inhibiting its concentration in an animal study. 
- The study of the broadband NIRS measurements on the different age, gender groups to gain certain knowledge on the behavior of NIRS light through the scalp, skin, cerebral spinal fluid (CSF) and the brain in the human head.

- Optimization of the set of wavelengths that can be used in mNIRS to measure CCO changes in adults.

- Examine the effects of different sedation drugs administered intravenously on cerebral hemoglobin oxygen saturation during ventricular pacing of the TAVI procedure. 


\section{References:}

[1]. Ferrari, M., \& Quaresima, V., "Near Infrared Brain and Muscle Oximetry: From the Discovery to Current Applications", Journal of Near Infrared Spectroscopy, 20(1), 1-14 (2012).

[2]. Cope M: The Development of a Near-Infrared Spectroscopy System and Its Application for Noninvasive Monitoring of Cerebral Blood and Tissue Oxygenation in the Newborn infant. University College London; 1991.

[3] Michel, H., Behr, J., Harrenga, A., \& Kannt, A., "CYTOCHROME C OXIDASE: Structure and Spectroscopy", Annual Review of Biophysics And Biomolecular Structure, 27(1), 329356 (1998).

[4] Wang, L., \& Wu, H., "Biomedical optics". Hoboken, N.J.: Wiley-Interscience, (2007).

[5] Steenhaut, K., BOVE, T., De Hert, S., \& MOERMAN, A. "Comparison of three NIRS devices for the measurement of microvascular reactivity" (2012).

[6] Mehnert, Jan., "Evaluation and Optimization of Recent Analytical Approaches for In-Vivo Measurements with Near-Infrared Spectroscopy". Journal of biomolecular, 4(1),25-30, (2013).

[7] Jue, T., \& Masuda, K. "Application of near infrared spectroscopy in biomedicine", New York: Springer (2013).

[8] Torricelli, A., Contini, D., Pifferi, A., Caffini, M., Re, R., Zucchelli, L., \& Spinelli, L. ,"Time domain functional NIRS imaging for human brain mapping”, Neuroimage, 85, 28-50 (2014).

[9] Bae H., "Basic Principle and Practical Implementation of Near-Infrared Spectroscopy (NIRS)”. In: Lin YL., Kyung CM., Yasuura H., Liu Y. (eds) Smart Sensors and Systems. Springer, Cham (2015). 
[10] Akke Bakker, Brianne Smith, Philip Ainslie and Kurt Smith., "Near-Infrared Spectroscopy, Applied Aspects of Ultrasonography in Humans Philip Ainslie”, IntechOpen, (2012)

[11] Delpy DT, Cope M., "Near-infrared spectroscopy and imaging of living systems", Philos Trans: Biol Sci. 352(1354):649-59 (1997).

[12] Arridge, S. R., Cope, M., \& Delpy, D. T., "The theoretical basis for the determination of optical path-lengths in tissue: temporal and frequency analysis". Physics in medicine and biology, 37, 7, (March 1992), 1531-1560, (1992).

[13] Rolfe, P., "In vivo near-infrared spectroscopy", Annual Review of Biomedical Engineering $2,715-754(200)$.

[14] Al-Rawi, P. G., Smielewski, P., \& Kirkpatrick, P. J, (2001). "Evaluation of a Near-Infrared Spectrometer (NIRO 300) for the Detection of Intracranial Oxygenation Changes in the Adult Head", Stroke, 32, 11, (2492-2500) (2001).

[15] Hardison, R. (1998)., "Hemoglobins from bacteria to man: evolution of different patterns of gene expression”. The Journal of Experimental Biology, 201(1), 1099-1117 (1998).

[16] Murkin, J. and Arango, M., "Near-infrared spectroscopy as an index of brain and tissue oxygenation", British Journal of Anaesthesia, 103(1), i3-i13 (2009).

[17] Bale, G., Elwell, C., \& Tachtsidis, I., "From Jöbsis to the present day: a review of clinical near-infrared spectroscopy measurements of cerebral cytochrome-c-oxidase", Journal Of Biomedical Optics, 21(9), 091307 (2016).

[18] Mason, M., Nicholls, P., \& Cooper, C., "The steady-state mechanism of cytochrome c oxidase: redox interactions between metal centres", Biochemical Journal, 422(2), 237-246 (2009). 
[19] Kolyva, C., Ghosh, A., Tachtsidis, I., Highton, D., Cooper, C., Smith, M., \& Elwell, C., "Cytochrome c oxidase response to changes in cerebral oxygen delivery in the adult brain shows higher brain-specificity than haemoglobin” Neuroimage, 85, 234-244 (2014).

[20] D. Arifler et al., "Optimal wavelength combinations for near-infrared spectroscopic monitoring of changes in brain tissue hemoglobin and cytochrome c oxidase concentrations," Biomed. Opt. Express 6(3), 933 (2015).

[21] Cooper, CE., Cope, M., Springett, R., Amess, PN., Penrice, J., and Tyszczuk, L., "Use of mitochondrial inhibitors to demonstrate that cytochrome oxidase near-infrared spectroscopy can measure mitochondrial dysfunction noninvasively in the brain", J Cereb Blood Flow Metab 19(1), 27-38 (1999).

[22] Geocadin, R., Koenig, M., Jia, X., Stevens, R., and Peberdy, M., "Management of Brain Injury After Resuscitation From Cardiac Arrest", Neurologic Clinics 26(2), 487-506 (2008).

[23] Frohlich, G., Lyon, R., Sasson, C., Crake, T., Whitbread, M., Indermuehle, A., Timmis, A., and Meier, P., "Out-of-Hospital Cardiac Arrest-Optimal Management", Current Cardiology Reviews 9(4), 316-324 (2014).

[24] Cournoyer, A., Iseppon, M., Chauny, J., Denault, A., Cossette, S., and Notebaert, É., "Nearinfrared Spectroscopy Monitoring During Cardiac Arrest: A Systematic Review and Metaanalysis", Academic Emergency Medicine 23(8), 851-862 (2016).

[25] Seeley, R., VanPutte, C., Regan, J., \& Russo, A. “Anatomy \& physiology”. New York: McGraw-Hill (2011).

[26] Papademetriou, M.D.; (2011) Multichannel near infrared spectroscopy to monitor cerebral oxygenation in infants and children supported in extracorporeal membrane oxygenation (ECMO). Doctoral thesis, UCL (University College London) (2011). 
[27] C. Cooper and R. Springett, "Measurement of cytochrome oxidase and mitochondrial energetics by near-infrared spectroscopy," Philosophical Transactions of the Royal Society B: Biological Sciences 352, 669-676 (1997).

[28] M. Kohl-Bareis, H. Obrig, J. Steinbrink, J. Malak, K. Uludag, and A. Villringer, "Noninvasive monitoring of cerebral blood flow by a dye bolus method: Separation of brain from skin and skull signals,” J. Biomed. Opt. 7, 464-470 (2002).

[29] J. Steinbrink, H. Wabnitz, H. Obrig, A. Villringer, and H. Rinneberg, "Determination of changes in NIR absorption using a layered model of the human head," Phys. Med. Biol. 46, 879-896 (2001).

[30] J. Choi, M. Wolf, V. Toronov, U. Wolf, C. Polzonetti, D. Hueber, L. Safonova, R. Gupta, A. Michalos, W. Mantulin, and E. Gratton, "Noninvasive determination of optical properties of adult brain: near-infrared spectroscopy approach,” J. Biomed. Opt. 9, 221-229 (2004).

[31] Saager, R., \& Berger, A., "Direct characterization and removal of interfering absorption trends in two-layer turbid media" Journal Of The Optical Society Of America A, 22(9), 1874 (2005).

[32] Matcher, S., Cope, M., \& Delpy, D., "Use of the water absorption spectrum to quantify tissue chromophore concentration changes in near-infrared spectroscopy", Physics In Medicine And Biology, 39(1), 177-196 (1994).

[33] Hunter, R., Patterson, M., Farrell, T., \& Hayward, J., "Haemoglobin oxygenation of a twolayer tissue-simulating phantom from time-resolved reflectance: effect of top layer thickness", Physics in Medicine And Biology, 47(2), 193-208 (2002). 
[34] Okada, E. \& D.T. Delpy., "Near-infrared light propagation in an adult head model. I. Modeling of low-level scattering in the cerebrospinal fluid layer", Applied Optics 42, 29062914 (2003).

[35] Rakhshani Fatmehsari, Y., \& Moussavi, Z., "Designing and Implementing a Portable NearInfrared Imaging System for Monitoring of Human's Functional Brain Activity”, Journal Of Medical Devices. 4(1), (2015)

[36] S.J. Madsen., "Optical Methods and Instrumentation in Brain Imaging and Therapy", Bioanalysis, 3, (2013).

[37] Ebinger, M., "Core Topics in Neuroanesthesia and Neurointensive Care", Archives Of Neurology, 69(6) (2012).

[38] Al-Rawi, P \& Kirtpatrick, P., "Near infrared spectroscopy in carotid endarterectomy. Carotid Disease: The Role of Imaging in Diagnosis and Management”, Cambridge: Cambridge University Press, 372(2) (2007).

[39] Bhatt, M., Ayyalasomayajula, K., \& Yalavarthy, P., "Generalized Beer-Lambert model for near-infrared light propagation in thick biological tissues", Journal Of Biomedical Optics, 21(7), 076012 (2016).

[40] Teng, Y., Ding, H., Huang, L., Li, Y., Shan, Q., \& Ye, D. et al., "Non-invasive measurement and validation of tissue oxygen saturation covered with overlying tissues", Progress In Natural Science, 18(9), 1083-1088 (2008).

[41] Strangman,G., Franceschini M, A ., and Boas, D A., "Factors affecting the accuracy of nearinfrared spectroscopy concentration calculations for focal changes in oxygenation parameters", Neuroimage, 18 865-79 (2003). 
[42] Scholkmann, F., \& Wolf, M., "General equation for the differential pathlength factor of the frontal human head depending on wavelength and age", Journal Of Biomedical Optics, 18(10), 105004 (2013).

[43] Obrig $\mathrm{H}$ and Villringer A.,"Beyond the visible-imaging the human brain with light $\mathrm{J}$. Cereb", Blood Flow Metab. 23 1-18(2003).

[44] Groenhuis R.A., Ferwerda H.A., and Ten Bosch J.J., "Scattering and absorption of turbid materials determined from reflection measurements.” Applied Optics 22, 2456 (1983).

[45] Kim J.G., Xia M., and Liu H., "Extinction coefficients of hemoglobin for near infrared spectroscopy of tissue," IEEE Engineering in Medicine and Biology Magazine: The Quarterly Magazine of the Engineering in Medicine \& Biology Society 24, 118-121(2005).

[46] MacLeod, D., Ikeda, K., Vacchiano, C., Lobbestael, A., Wahr, J. and Shaw, A., “”, Journal of Cardiothoracic and Vascular Anesthesia, 26(6), 1007-1014 (2012).

[47] Taga, G., Homae, F., \& Watanabe, H., "Effects of source-detector distance of near infrared spectroscopy on the measurement of the cortical hemodynamic response in infants", Neuroimage, 38(3), 452-460 (2007).

[48] Yoshitani K, Kawaguchi M, Miura N, et al, "Effects of hemoglobin concentration, skull thickness, and the area of the cerebrospinal fluid layer on near-infrared spectroscopy measurements" Anesthesiology, 106, (2007);

[49] Davie, S., \& Grocott, H., "Impact of Extracranial Contamination on Regional Cerebral Oxygen Saturation”, Anesthesiology, 116(4), 834-840 (2012).

[50] Hyttel-Sorensen, S., Hessel, T. W., \& Greisen, G., "Peripheral tissue oximetry: comparing three commercial near-infrared spectroscopy oximeters on the forearm", Journal of Clinical Monitoring and Computing, 28(2), 149-155 (2014). 
[51] Fischer, G. W., \& Silvay, G., "Cerebral oximetry in cardiac and major vascular surgery”, HSR Proceedings in Intensive Care \& Cardiovascular Anesthesia, 2(4), 249-256 (2010).

[52] A. P. Tarasov, A. I. Egorov, D. A. Rogatkin, "Study of extracerebral contamination for three cerebral oximeters by Monte Carlo simulation using CT data," Proc. SPIE 10411, Clinical and Preclinical Optical Diagnostics, (2017).

[53] Hideo, E., "Measurement of brain activity by near infrared light", Journal of the National Institute of Information and Communications Technology, 51,73-88 (2004).

[54] Kocsis, L., Herman, P., \& Eke, A., “The modified Beer-Lambert law revisited”. Physics in Medicine And Biology, 51(5), 91-98 (2006).

[55] Pellicer, A., \& Bravo, M., "Near-infrared spectroscopy: A methodology-focused review”, Seminars In Fetal And Neonatal Medicine, 16(1), $42-49$ (2011).

[56] Kohl, M., Lindauer, U., Dirnagl, U., and Villringer. A., "Separation of changes in light scattering and chromophore concentrations during cortical spreading depression in rats" Opt. Lett. 23 555-7 (1998).

[57] C. Sato, M. Nemoto and M. Tamura, "Reassessment of Activity-Related Optical Signals in Somatosensory Cortex by an Algorithm with Wavelength-Dependent Path Length.," The Japanese Journal of Physiology 52, 301-312 (2002).

[58] Fantini, S., Franceschini, M., \& Gratton, E., "Semi-infinite-geometry boundary problem for light migration in highly scattering media: a frequency-domain study in the diffusion approximation”. Journal Of The Optical Society Of America B, 11(10), 2128 (1994). 
[59] A. Kienle and M. S. Patterson, "Improved solutions of the steady-state and the time-resolved diffusion equations for reflectance from a semi-infinite turbid medium,” J. Opt. Soc. Am. A 14(1), 246-254 (1997).

[60] Yeganeh, H. Z., Toronov, V., Elliott, J. T., Diop, M., Lee, T. Y., and St. Lawrence, K. "Broadband continuous-wave technique to measure baseline values and changes in the tissue chromophore concentrations,” Biomed. Opt Express,11(3), 2761-2770 (2012).

[61] Pucci, O., Toronov, V., \& St. Lawrence, K., "Measurement of the optical properties of a two-layer model of the human head using broadband near-infrared spectroscopy". Applied Optics, 49(32), $6324(2010)$

[62] Nosrati, R., Lin, S., Ramadeen, A., Monjazebi, D., Dorian, P., and Toronov, V., "Cerebral Hemodynamics and Metabolism During Cardiac Arrest and Cardiopulmonary Resuscitation Using Hyperspectral Near Infrared Spectroscopy", Circulation Journal 81(6), 879-887 (2017).

[63] Matcher S. J., Elwell C. E., Cooper C. E., Cope M., Delpy D. T., "Performance comparison of several published tissue near-infrared spectroscopy algorithms," Anal. Biochem. 227(1), 54-68 (1995).

[64] Grossweiner, L., Jones, L., Grossweiner, J., \& Rogers, B. "The science of phototherapy”, Dordrecht: Springer. 143-170 (2005)

[65] Hoshi, Y., Shimada, M., Sato, C., and Iguchi, Y., "Reevaluation of near-infrared light propagation in the adult human head: implications for functional near-infrared spectroscopy", Journal of Biomedical Optics 10(6), 064032 (2005).

[66] Sanderson, T., Wider, J., Lee, I., Reynolds, C., Liu, J., Lepore, B., Tousignant, R., Bukowski, M., and JohntSOn, H. et al., "Inhibitory modulation of cytochrome c oxidase activity with 
specific near-infrared light wavelengths attenuates brain ischemia/reperfusion injury", Scientific Reports 8(1) (2018).

[67] Tisdall, M., Tachtsidis, I., Leung, T., Elwell, C., and Smith, M., "Near-infrared spectroscopic quantification of changes in the concentration of oxidized cytochrome c oxidase in the healthy human brain during hypoxemia", Journal of Biomedical Optics 12(2), 024002 (2007).

[68] Tisdall, M., Tachtsidis, I., Leung, T., Elwell, C., and Smith, M., "Increase in cerebral aerobic metabolism by normobaric hyperoxia after traumatic brain injury", Journal of Neurosurgery 109(3), 424-432 (2008).

[69] Jobsis, F., "Noninvasive, infrared monitoring of cerebral and myocardial oxygen sufficiency and circulatory parameters", Science 198(4323), 1264-1267 (1977).

[70] Bale, G., Elwell, C., and Tachtsidis, I., "From Jöbsis to the present day: a review of clinical near-infrared spectroscopy measurements of cerebral cytochrome-c-oxidase", Journal of Biomedical Optics 21(9), 091307 (2016).

[71] Thiele, R., Ikeda, K., Wang, Y., Bartz, R., and Zuo, Z., "Broadband near-infrared spectroscopy can detect cyanide-induced cytochrome aa3 inhibition in rats: a proof of concept study", Canadian Journal of Anesthesia/Journal canadien d'anesthésie 64(4), 376384 (2016).

[72] Ferrari, M., and Quaresima, V., "A brief review on the hitSOry of human functional nearinfrared spectroscopy (fNIRS) development and fields of application", Neuroimage 63(2), 921-935 (2012). 
[73] Kakihana, Y., Matsunaga, A., Yasuda, T., Imabayashi, T., Kanmura, Y., and Tamura, M., "Brain oxymetry in the operating room: current status and future directions with particular regard to cytochrome oxidase", Journal of Biomedical Optics 13(3), 033001 (2008).

[74] Okada, E., and Delpy, D., "Near-infrared light propagation in an adult head model II Effect of superficial tissue thickness on the sensitivity of the near-infrared spectroscopy signal", Applied Optics 42(16), 2915 (2003).

[75] Germon, T., Young, A., Manara, A., and Nelson, R., "Extracerebral absorption of near infrared light influences the detection of increased cerebral oxygenation monitored by near infrared spectroscopy.", Journal of Neurology, Neurosurgery \& Psychiatry 58(4), 477-479 (1995).

[76] Smith, M., and Elwell, C., "Near-Infrared Spectroscopy: Shedding Light on the Injured Brain", Anesthesia \& Analgesia 108(4), 1055-1057 (2009).

[77] Greenberg, DL., Fetzer, DL., and Payne, ME., "Aging, gender, and the elderly adult brain: an examination of analytical strategies", Neurobiology of aging 29(2), 290-302 (2008). 77

[78] Resnick, SM., Pham, DL., Kraut, MA., Zonderman, AB., and Davatzikos C., "Longitudinal magnetic resonance imaging studies of older adults: A shrinking brain", J Neurosci 23(1), 3295-3301(2003).

[79] Kleinschmidt, A., Obrig, H., Requardt, M., Merboldt, K., Dirnagl, U., Villringer, A., and Frahm, J., "Simultaneous Recording of Cerebral Blood Oxygenation Changes during Human Brain Activation by Magnetic Resonance Imaging and Near-Infrared Spectroscopy", Journal of Cerebral Blood Flow \& Metabolism 16(5), 817-826 (1996). 
[80] Wabnitz, H., Moeller, M., Liebert, A., Obrig, H., Steinbrink, J., and Macdonald, R., " Timeresolved near-infared spectroscopy and imaging of the adult human brain", Advances in Experimental Medicine and Biology 662(1), 143-148 (2009).

[81] Thirumala, P. D., Muluk, S., Udesh, R., Mehta, A., Schindler, J., Mulukutla, S., ... Gleason, T. (2017). Carotid Artery Disease and Periprocedural Stroke Risk after Transcatheter Aortic Valve Implantation. Annals of Cardiac Anaesthesia, 20(2), 145-151.

[82] Molinari, F., Liboni, W., Grippi, G., \& Negri, E. (2006). Relationship between oxygen supply and cerebral blood flow assessed by transcranial Doppler and near - infrared spectroscopy in healthy subjects during breath - holding. Journal of NeuroEngineering and Rehabilitation, 3, 16.

[83] Toronov, Vladislav Y., Xiaofeng Zhang, and Andrew G. Webb. "Near-Infrared Study of the Underlying Physiology of the Functional Magnetic Resonance Signal in Humans during Hypoxia." Proceedings of SPIE--the International Society for Optical Engineering 5686 (2005): 543-546. PMC. Web. 10 Aug. 2018.

[84] L. Holper, F. Scholkmann, and E. Seifritz, "Time-frequency dynamics of the sum of intraand extracerebral hemodynamic functional connectivity during resting-state and respiratory challenges assessed by multimodal functional near-infrared spectroscopy," NeuroImage 120, 481-492 (2015).

[85] Holper, L., and Mann, J., "Test-retest reliability of brain mitochondrial cytochrome-coxidase assessed by functional near-infrared spectroscopy", Journal of Biomedical Optics 23(05), 1 (2018).

[86] Young, A., Germon, T., Barnett, N., Manara, A., and Nelson, R., "Behaviour of near-infrared light in the adult human head: implications for clinical near-infrared spectroscopy", British Journal of Anaesthesia 84(1), 38-42 (2000). 
87. Nomura, F., Naruse, H., Duplessis, A., Hiramatsu, T., Forbess, J., Holtzman, D., Volpe, J., Jonas, R., and Tsuji, M., "Cerebral Oxygenation Measured by Near Infrared Spectroscopy during Cardiopulmonary Bypass and Deep Hypothermic Circulatory Arrest in Piglets", Pediatric Research. 40(6), 790-796 (1996).

88. Schewe, JC., Thudium, MO., Kappler, J., Steinhagen, F., Eichhorn, L.,and Erdfelder, F., " Monitoring of cerebral oxygen saturation during resuscitation in out-of-hospital cardiac arrest: A feasibility study in a physician staffed emergency medical system", Scand J Trauma Resusc Emerg Med. 22(15),58 (2014).

89.Frisch, A., Suffoletto, BP., Frank, R., Martin-Gill, C., and Menegazzi, JJ., "Potential utility of near-infrared spectroscopy in out-of-hospital cardiac arrest: An illustrative case series", Prehospital Emerg Care. 16(1), 564-570 (2012).

90. Miceli, A., Gilmanov, D., Murzi, M., Marchi, F., Ferrarini, M., Cerillo, A., Quaini, E., Solinas, M., and Berti, S. et al., "Minimally invasive aortic valve replacement with a sutureless valve through a right anterior mini-thoracotomy versus transcatheter aortic valve implantation in high-risk patients", European Journal of Cardio-Thoracic Surgery. 49(3), 960-965 (2015). 